\title{
ASYMPTOTIC BEHAVIOR FOR A DISSIPATIVE NONLINEAR SCHRÖDINGER EQUATION
}

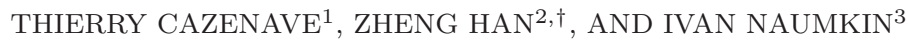

\begin{abstract}
We consider the Schrödinger equation with nonlinear dissipation
$i \partial_{t} u+\Delta u=\lambda|u|^{\alpha} u$

in $\mathbb{R}^{N}, N \geq 1$, where $\lambda \in \mathbb{C}$ with $\Im \lambda<0$. Assuming $\frac{2}{N+2}<\alpha<\frac{2}{N}$, we give a precise description of the long-time behavior of the solutions (including decay rates in $L^{2}$ and $L^{\infty}$, and asymptotic profile), for a class of arbitrarily large initial data.
\end{abstract}

\section{InTRODUCTION}

In this paper, we consider the following nonlinear Schrödinger equation

$$
\left\{\begin{array}{l}
i \partial_{t} u+\Delta u=\lambda|u|^{\alpha} u \\
u(0, x)=u_{0}
\end{array}\right.
$$

where $\lambda \in \mathbb{C}$ with

$$
\Im \lambda<0,
$$

and $0<\alpha<\frac{2}{N}$. This equation is a particular case of the complex Ginzburg-Landau equation on $\mathbb{R}^{N}$

$$
\partial_{t} u=e^{i \theta} \Delta u+\zeta|u|^{\alpha} u
$$

where $|\theta| \leq \frac{\pi}{2}$ and $\zeta \in \mathbb{C}$, which in turn is a generic modulation equation that decribes the nonlinear evolution of patterns at near-critical conditions. See for instance $[20,9,17]$.

If $\alpha<\frac{4}{N}$, then equation (NLS) is mass-subcritical, hence under assumption (1.1) the associated initial value problem is globally well-posed in $L^{2}\left(\mathbb{R}^{N}\right)$ and in $H^{1}\left(\mathbb{R}^{N}\right)$. See e.g. [2, Proposition 2.1].

For the large-time behavior of the solutions, the exponent $\alpha=\frac{2}{N}$ is critical. More precisely, if $\alpha>\frac{2}{N}, \lambda \in \mathbb{C}$, then for a large set of initial values the corresponding solutions scatter as $t \rightarrow \infty$, i.e. they behave like the solutions of the free Schrödinger equation. See $[21,11,12,7,10,18,1,5]$. On the other hand, if $\alpha \leq \frac{2}{N}$ and (1.1) holds then, due to the dissipative nature of the nonlinear term, the solutions of equation (NLS) often decay faster compared to the solutions of the free Schrödinger equation. In particular, in the case $\alpha=\frac{2}{N}$, a large class of solutions of (NLS) have the decay rate $(t \log t)^{-\frac{N}{2}}$ as $t \rightarrow \infty$, see $[19,15,16,6]$. It is worth noting that, as proved in [6], the limit

$$
\lim _{t \rightarrow \infty}(t \log t)^{\frac{N}{2}}\|u(t)\|_{L^{\infty}}=(\alpha|\Im \lambda|)^{-\frac{N}{2}}
$$

1991 Mathematics Subject Classification. Primary 35Q55; secondary 35B40.

Key words and phrases. Nonlinear Schrödinger equation; dissipative nonlinearity; Asymptotic behavior.

$\dagger$ Corresponding author.

Zheng Han thanks NSFC 11671353,11401153, Zhejiang Provincial Natural Science Foundation of China under Grant No. LY18A010025, and CSC for their financial support.

Ivan Naumkin is a Fellow of Sistema Nacional de Investigadores. He was partially supported by project PAPIIT IA101820. 
exists and is independent of the initial value (for a certain class of solutions).

In the case $\alpha<\frac{2}{N}$, it is possible (still assuming (1.1)) to derive strong a priori estimates for the solutions to (NLS) under some further "dissipative" condition on $\lambda$. These estimates are then used to describe the large-time behavior of the solutions to (NLS) for $\alpha<\frac{2}{N}$ sufficiently close to the critical power $\frac{2}{N}$. More precisely, in the one-dimensional case $N=1$, if $\alpha<2$ is sufficiently close to 2 and under the dissipative condition

$$
\frac{\alpha}{2 \sqrt{\alpha+1}}|\Re \lambda| \leq|\Im \lambda|,
$$

the large-time asymptotic behavior of solutions is described in [16] for any initial value in $H^{1}(\mathbb{R}) \cap L^{2}\left(\mathbb{R},|x|^{2} d x\right)$. In particular, the solutions satisfy

$$
\|u(t)\|_{L^{\infty}} \lesssim t^{-\frac{1}{\alpha}}
$$

In addition, it is proved in [13] that for any space dimension $N \geq 1$, under the dissipative condition (1.2) and for $\alpha<\frac{2}{N}$ sufficiently close to $\frac{2}{N}$, all solutions with initial value in $H^{1}\left(\mathbb{R}^{N}\right) \cap L^{2}\left(\mathbb{R}^{N},|x|^{2} d x\right)$ satisfy the $L^{2}$-decay estimate

$$
\|u(t)\|_{L^{2}} \lesssim t^{-\left(\frac{1}{\alpha}-\frac{N}{2}\right) q}
$$

for all $q<\frac{2}{N+2}, q \leq \frac{1}{2}$. If there is no "dissipative" condition on $\lambda$, it seems problematic to derive a priori estimates for all solutions. Therefore, in order to study the large-time behavior of the solutions, further assumptions on the initial data are required. This is achieved in $[15,14]$, where the dissipative condition (1.2) is removed and the time decay estimates (1.3), as well as lower estimates are established, and the large-time asymptotic behavior of the solutions is described, in the case of space dimensions $N=1,2,3$, for all $\alpha<\frac{2}{N}$ sufficiently close to $\frac{2}{N}$, but only for all sufficiently small initial data in a certain space. In the case of any space dimension $N \geq 1$, the large-time asymptotic behavior of solutions is studied in [2], for $\alpha<\frac{2}{N}$ sufficiently close to $\frac{2}{N}$ and for a class of arbitrarily large nonvanishing initial values. (A nonvanishing condition appears due to the lack of regularity of the nonlinear term, see [6].) Note that it is proved in [2] that, for the solutions studied there, the limit

$$
\lim _{t \rightarrow \infty} t^{\frac{1}{\alpha}}\|u(t)\|_{L^{\infty}}=\left(\frac{2 \alpha|\Im \lambda|}{2-N \alpha}\right)^{-\frac{1}{\alpha}}
$$

exists and is independent of the initial value, similarly to what happens in the case $\alpha=\frac{2}{N}$. Again, since (1.2) is also not used in [2], the results are obtained only for a class of initial data (not necessarily small).

The aim of the present work is to complete the previous results on the timeasymptotic behavior of the solutions obtained in $[2,13,14,16]$. More precisely, we do not impose any condition on $\lambda$ other than (1.1), and we improve the lower conditions on $\alpha$, replacing certain relative smallness assumptions by the explicit condition

$$
\frac{2}{N+2}<\alpha<\frac{2}{N}
$$

Before stating our results, we introduce the function spaces we will use. We fix three integers $k, m, n$ sufficiently large so that

$$
\begin{gathered}
k>\frac{N}{2}+4, \\
n>\max \left\{\frac{20}{\alpha^{2}}, \frac{N(2-N \alpha)(k+4)}{\alpha}, \frac{2 N(k+2)(2-N \alpha)}{(N+2) \alpha-2}\right\}, \\
m>\max \left\{\frac{k+n+1}{2}, \frac{5 n \alpha|\lambda|(1+\alpha|\Im \lambda|)}{N(2-N \alpha)|\Im \lambda|}\right\},
\end{gathered}
$$


and we let

$$
J=2 m+2+k+n .
$$

Moreover, we define the nonincreasing function $M:\{0, \cdots, J\} \rightarrow\{0, \cdots, n\}$ by

$$
M(p)= \begin{cases}n & 0 \leq p \leq J-n, \\ J-p & J-n \leq p \leq J .\end{cases}
$$

We introduce the Banach space $\mathcal{X}$ defined in $[6,5]$

$$
\begin{gathered}
\mathcal{X}=\left\{u \in H^{J}\left(\mathbb{R}^{N}\right) ;\langle x\rangle^{n} D^{\beta} u \in L^{\infty}\left(\mathbb{R}^{N}\right) \text { for } 0 \leq|\beta| \leq 2 m,\right. \\
\left.\langle x\rangle^{M(|\beta|)} D^{\beta} u \in L^{2}\left(\mathbb{R}^{N}\right) \text { for } 2 m+1 \leq|\beta| \leq J\right\}
\end{gathered}
$$

and we equip $\mathcal{X}$ with the norm

$$
\|u\|_{\mathcal{X}}=\sup _{0 \leq|\beta| \leq 2 m}\left\|\langle\cdot\rangle^{n} D^{\beta} u\right\|_{L^{\infty}}+\sup _{2 m+1 \leq|\beta| \leq J}\left\|\langle\cdot\rangle^{M(|\beta|)} D^{\beta} u\right\|_{L^{2}}
$$

where

$$
\langle x\rangle=\left(1+|x|^{2}\right)^{\frac{1}{2}} .
$$

As observed above, under assumptions (1.1) and (1.4), the initial value problem associated with equation (NLS) is globally well-posed in $H^{1}\left(\mathbb{R}^{N}\right)$. Our main result is the following.

Theorem 1.1. Let $\lambda \in \mathbb{C}$ with $\Im \lambda<0$, and let $\alpha$ satisfy (1.4). Assume (1.5)-(1.7) and let $\mathcal{X}$ be defined by (1.10). Let $v_{0} \in \mathcal{X}$ satisfy

$$
\inf _{x \in \mathbb{R}^{N}}\langle x\rangle^{n}\left|v_{0}(x)\right|>0
$$

Given $b \in \mathbb{R}$, let $u_{0} \in H^{1}\left(\mathbb{R}^{N}\right)$ be given by $u_{0}(x)=e^{i \frac{b|x|^{2}}{4}} v_{0}(x)$ and let $u \in$ $C\left([0, \infty), H^{1}\left(\mathbb{R}^{N}\right)\right)$ be the corresponding solution of (NLS). If b is sufficiently large, then $u \in L^{\infty}\left((0, \infty) \times \mathbb{R}^{N}\right) \cap L^{\infty}\left((0, \infty), H^{1}\left(\mathbb{R}^{N}\right)\right)$, and there exist $C, \delta>0$, and $f_{0}, \omega_{0} \in L^{\infty}\left(\mathbb{R}^{N}\right) \cap C\left(\mathbb{R}^{N}\right)$ with $\left\|f_{0}\right\|_{L^{\infty}} \leq \frac{1}{2}$ and $\langle\cdot\rangle^{n} \omega_{0} \in L^{\infty}\left(\mathbb{R}^{N}\right)$ such that

$$
t^{\frac{1}{\alpha}-\frac{N}{2}}\|u(t, \cdot)-z(t, \cdot)\|_{L^{2}}+t^{\frac{1}{\alpha}}\|u(t, \cdot)-z(t, \cdot)\|_{L^{\infty}} \leq C t^{-\delta}
$$

for $t \geq 1$, where

$$
z(t, x)=(1+b t)^{-\frac{N}{2}} e^{i \Theta(t, x)} \Psi\left(t, \frac{x}{1+b t}\right) \omega_{0}\left(\frac{x}{1+b t}\right)
$$

with

and

$$
\Theta(t, x)=\frac{b|x|^{2}}{4(1+b t)}-\frac{\Re \lambda}{\Im \lambda} \log \Psi\left(t, \frac{x}{1+b t}\right)
$$

$$
\Psi(t, y)=\left(\frac{1+f_{0}(y)}{1+f_{0}(y)+\frac{2 \alpha|\Im \lambda|}{b(2-N \alpha)}\left|v_{0}(y)\right|^{\alpha}\left[(1+b t)^{\frac{2-N \alpha}{2}}-1\right]}\right)^{\frac{1}{\alpha}} .
$$

Moreover,

$$
\left|\omega_{0}\right|^{\alpha}=\frac{\left|v_{0}\right|^{\alpha}}{1+f_{0}}
$$

so that $\frac{3}{2}\left|v_{0}\right|^{\alpha} \leq\left|\omega_{0}\right|^{\alpha} \leq 2\left|v_{0}\right|^{\alpha}$. In addition,

$$
t\|u\|_{L^{\infty}}^{\alpha} \underset{t \uparrow \frac{1}{b}}{\longrightarrow} \frac{2-N \alpha}{2 \alpha|\Im \lambda|}
$$

and

$$
a \leq(1+b t)^{\left(\frac{1}{\alpha}-\frac{N}{2}\right)\left(1-\frac{N}{2 n}\right)}\|u(t)\|_{L^{2}} \leq A,
$$

as $t \rightarrow \infty$, for some constants $0<a \leq A<\infty$.

Remark 1.2. Here are some comments on Theorem 1.1. 
(i) Theorem 1.1 is valid in any dimension $N \geq 1$, and for any $\lambda \in \mathbb{C}$ with $\Im \lambda<0$. The main restrictions are that the initial value $u_{0}$ must be sufficiently smooth, bounded from below in the sense (1.11), and oscillatory in the sense that $b$ must be sufficiently large. On the other hand, there is no restriction on the amplitude of $u_{0}$.

(ii) A typical initial value which is admissible in Theorem 1.1 is $v_{0}=c\langle\cdot\rangle^{-n}+\varphi$ with $c \in \mathbb{C}, c \neq 0$, and $\varphi \in \mathcal{S}\left(\mathbb{R}^{N}\right),|\varphi| \leq(|c|-\varepsilon)\langle\cdot\rangle^{-n}, \varepsilon>0$.

(iii) The limit (1.13) gives the exact decay rate of $\|u(t)\|_{L^{\infty}}$, and this limit is independent of the initial value $u_{0}$. Compare [2, Remark 1.2 (iv)].

(iv) Estimate (1.14) shows that $\|u(t)\|_{L^{2}}$ is equivalent as $t \rightarrow \infty$ to $t^{-\left(\frac{1}{\alpha}-\frac{N}{2}\right)\left(1-\frac{N}{2 n}\right)}$. In particular, we see that the decay rate of $\|u(t)\|_{L^{2}}$ depends on the initial value, through the parameter $n$ which can be chosen (provided it is sufficiently large to satisfy (1.6)).

(v) Since

$$
\liminf _{t \rightarrow \infty} t^{\frac{1}{\alpha}}\|u(t)\|_{L^{\infty}}>0, \quad t^{\frac{1}{\alpha}-\frac{N}{2}}\|u(t)\|_{L^{2}} \underset{t \rightarrow \infty}{\longrightarrow} \infty,
$$

by (1.13)-(1.14), we see that the asymptotic behavior of $u(t)$ as $t \rightarrow \infty$ is described by the asymptotic estimate (1.12).

(vi) We do not know if the lower condition in (1.4) on the power $\alpha$ is necessary to derive the asymptotic expansion (1.12). However, assumption (1.4) plays a crucial role in the proof of Proposition 4.1 below to control $\left\|\frac{\Delta v}{|v|}\right\|_{L^{\infty}}$ (see (4.52)), which in turn is used in the proof of Proposition 5.1 to prove that $f(t)$ is convergent to $f_{0}$ in $L^{\infty}\left(\mathbb{R}^{N}\right)$ as $t \uparrow \frac{1}{b}$. Condition (1.4) is also essential to establish (5.24) in the proof of Proposition 5.1.

The general strategy we use to prove Theorem 1.1 is inspired by $[6,5]$. In order to obtain our results, we need strong decay and regularity of the initial data. As the nonlinearity $|u|^{\alpha} u$ can be not smooth enough, we require the nonvanishing condition (1.11) as well (see [6] for a discussion on this regularity issue). This explains the space $\mathcal{X}$ we work with. The other main ingredient in the strategy of $[6,5]$ is the application of the pseudo-conformal transformation, which is given by

$$
v(t, x)=(1-b t)^{-\frac{N}{2}} u\left(\frac{t}{1-b t}, \frac{x}{1-b t}\right) e^{-i \frac{b|x|^{2}}{4(1-b t)}}, \quad t \geq 0, x \in \mathbb{R}^{N},
$$

for any $b>0$. Using this transformation, we see that the equation (NLS) is equivalent to the nonautonomous equation

$$
\left\{\begin{array}{l}
i \partial_{t} v+\Delta v=\lambda(1-b t)^{-\frac{4-N \alpha}{2}}|v|^{\alpha} v \\
v(0, x)=v_{0}
\end{array}\right.
$$

The last equation reveals the main issue that appears in the case when $\alpha \leq \frac{2}{N}$ : the factor $(1-b t)^{-\frac{4-N \alpha}{2}}$ is not integrable at $t=1 / b$. In order to deal with this problem, in the critical case $\alpha=\frac{2}{N}$ considered in [5], the solution $v$ is estimated by allowing a certain growth of the norms appearing in $\mathcal{X}$. Then, by the Duhamel's formula for $\left(\mathrm{NLS}_{b}\right)$

$$
v(t)=e^{i t \Delta} v_{0}+\lambda \int_{0}^{t}(1-b s)^{-\frac{4-N \alpha}{2}} e^{i(t-s) \Delta}|v(s)|^{\alpha} v(s) d s
$$

and the elementary inequality

$$
\int_{0}^{t}(1-b s)^{-1-\nu} d s=\frac{1}{b \nu}\left((1-b t)^{-\nu}-1\right) \leq \frac{1}{b \nu}(1-b t)^{-\nu},
$$

if $e^{i(t-s) \Delta}|v(s)|^{\alpha}(s)$ is estimated in a certain norm by $(1-b s)^{-\mu}$, the solution $v$ is controlled in the same norm by $(1-b s)^{-\mu-\frac{2-N \alpha}{2}}$. In the case when $\alpha=\frac{2}{N}, v$ is 
controlled by the same power $(1-b s)^{-\mu}$ and this is used in [5] to close appropriate estimates. In the case $\alpha<\frac{2}{N}$, there appears an extra singularity $(1-b s)^{-\frac{2-N \alpha}{2}}$ in the control of $e^{i(t-s) \Delta}|v(s)|^{\alpha} v(s)$. In [2], this problem is overcome by using the extra decay of the solution due to dissipation. Namely,

$$
v(s) \sim b \frac{2-N \alpha}{2 \alpha|\Im \lambda|}(1-b s)^{-\frac{2-N \alpha}{2 \alpha}}
$$

as $s \rightarrow 1 / b$ (see (3.22) below). Unfortunately, the price to pay for this extra decay is the factor $b$ in the right-hand side of (1.17), which makes it impossible to obtain smallness for large $b$ in the last term in (1.16), when one applies a contraction argument. One solution to this problem is to use the factor $2-N \alpha$ in (1.17), which is small if $\alpha$ is relatively close to the critical power $\alpha=2 / N$, to close the required estimates. This is done in [2]. In the present paper we remove this assumption and replace it by the condition (1.4). This requires two new ingredients. First of all, we observe that under certain assumptions, not only the solution itself has an extra decay. The derivatives as well enjoy this property (see Proposition 3.2 below). The second ingredient is to allow a very large growth of the derivatives of the solution as $s \rightarrow 1 / b$. Roughly speaking, we let the derivatives $\left|D^{\beta} v\right| \sim(1-b s)^{-|\beta| \sigma}$, when $s \rightarrow 1 / b$ (in the previous works [5, 2], all the derivatives of a given order $\beta$ behave as $(1-b s)^{-\sigma_{|\beta|}}$, for some $\left.\sigma_{|\beta|} \leq 1\right)$. Let us try to explain how we use these ingredients to establish the necessary estimates. Differentiating equation $\left(\mathrm{NLS}_{b}\right)$ and using the condition $\Im \lambda<0$, we deduce (see Proposition 2.1 below)

$$
\begin{aligned}
\left|D^{\beta} v\right| \leq & \left|D^{\beta} v_{0}\right| \\
& +\int_{0}^{t}\left|D^{\beta} v\right| d s+\left.C \sum_{\substack{\gamma_{1}+\gamma_{2}=\beta,\left|\gamma_{1}\right| \geq 1}} \int_{0}^{t}(1-b t)^{-\frac{4-N \alpha}{2}}\left|D^{\gamma_{1}}\right| v\right|^{\alpha}|| D^{\gamma_{2}} v \mid d s .
\end{aligned}
$$

One of the key observations is that due to the dissipation $\Im \lambda<0$, the term $(1-b t)^{-\frac{4-N \alpha}{2}}|v|^{\alpha}\left|D^{\beta} v\right|$ is absent from (1.18). Then, as far as we can control the derivatives $\left.\left|D^{\gamma_{1}}\right| v\right|^{\alpha} \mid$ by Proposition 3.2 below, we control the derivatives of the solution $D^{\beta} v$ without gaining extra singularity or loosing the smallness because of the large factor $b$. We must stop this argument at some stage because, as it can be observed from (3.32), the derivatives $\left.\left|D^{\gamma_{1}}\right| v\right|^{\alpha} \mid$ are estimated in terms of derivatives of higher-order $\left|\gamma_{1}\right|+2$, hence a loss of two derivatives. Letting the exact stage $\left|\gamma_{1}\right|=M$ at which we stop this argument unknown for a moment, we estimate the highest two derivatives by assuming that $\left|D^{\beta} v\right| \sim(1-b s)^{-|\beta| \sigma}$ and using the dissipative behavior (1.17). Then, the derivative $D^{\beta} \int_{0}^{t}(1-b s)^{-\frac{4-N \alpha}{2}} e^{i(t-s) \Delta}|v(s)|^{\alpha} v(s) d s$ in (1.16) is controlled by $\frac{C}{|\beta| \sigma}(1-b s)^{-|\beta| \sigma}$. Letting $|\beta|$ be sufficiently large, that is, letting $m$ be sufficiently large, we obtain a small factor $\frac{C}{|\beta| \sigma}$, which then is used to complete the estimates on the solution $v$ of $\left(\mathrm{NLS}_{b}\right)$.

Remark 1.3. Here are some comments on blowup in equation (NLS), when the condition (1.1) is not satisfied or when $\alpha \geq \frac{4}{N}$.

(i) If $\Im \lambda \geq 0$, then blowup may occur in equation (NLS). Indeed, if $\Im \lambda>0$, then finite-time blowup occurs for equation (NLS), at least for $H^{1}$-subcritical powers $(N-2) \alpha<4$. See [4, 3]. Moreover, if $\alpha<\frac{2}{N}$, then all nontrivial solutions blow up in finite or infinite time, see [1]. Finite-time blowup also occurs if $\Im \lambda=0, \Re \lambda<0$, and $\alpha \geq \frac{4}{N}$, since in this case (NLS) is the standard focusing nonlinear Schrödinger equation.

(ii) If $\Im \lambda<0, \alpha>\frac{4}{N}$ and condition (1.2) is not satisfied, then whether or not some solutions of (NLS) blow up in finite time seems to be an open question. 
The rest of this paper is organized as follows. In Section 2 we establish preliminary estimates, for the nonhomogeneous Schrödinger equation, and for derivatives of the form $D^{\beta}\left(|v|^{\rho}\right)$ where $v$ is a given function. In Section 3, we prove a priori estimates for certain solutions of $\left(\mathrm{NLS}_{b}\right)$. These estimates are used in Section 4 to prove global existence (i.e., on the time interval $\left[0, \frac{1}{b}\right)$ for certain solutions of $\left(\mathrm{NLS}_{b}\right)$. Finally, in Section 5, we describe the asymptotic behavior of these solutions as $t \rightarrow \frac{1}{b}$ and complete the proof of Theorem 1.1.

\section{Preliminary estimates}

We begin by proving estimates for the nonhomogeneous Schrödinger equation

$$
\left\{\begin{array}{l}
i \partial_{t} v+\Delta v=f, \\
v(0, x)=v_{0},
\end{array}\right.
$$

which are modified versions of estimates in [6, Proposition 2.1].

Proposition 2.1. Assume

$$
k>\frac{N}{2}+2, \quad n>\frac{N}{2}+1, \quad 2 m \geq k+n+1,
$$

(1.8), and let $\mathcal{X}$ be defined by (1.10). It follows that there exists a constant $A=$ $A(N, n, k, m)$ such that if $T>0, v_{0} \in \mathcal{X}$ and $f \in C([0, T], \mathcal{X})$, then for all $0 \leq t \leq T$ the solution $v$ of (2.1) satisfies the following estimates: if $|\beta| \leq 2 m-2$, then

$$
\begin{aligned}
\left|\langle x\rangle^{n} D^{\beta} v\right| \leq & \left\|\langle\cdot\rangle^{n} D^{\beta} v_{0}\right\|_{L^{\infty}}+\int_{0}^{t} \sup _{|\gamma| \leq|\beta|+2}\left\|\langle\cdot\rangle^{n} D^{\gamma} v(s)\right\|_{L^{\infty}} d s \\
& +\Im \int_{0}^{t} \frac{\langle x\rangle^{2 n} D^{\beta} f D^{\beta} \bar{v}}{\langle x\rangle^{n}\left|D^{\beta} v\right|} d s,
\end{aligned}
$$

for all $x \in \mathbb{R}^{N}$. If $2 m-1 \leq|\beta| \leq 2 m$, then

$$
\begin{aligned}
\left|\langle x\rangle^{n} D^{\beta} v\right| \leq & \left\|\langle\cdot\rangle^{n} D^{\beta} v_{0}\right\|_{L^{\infty}}+A \int_{0}^{t} \sup _{|\beta|+2 \leq|\gamma| \leq|\beta|+k+2}\left\|\langle\cdot\rangle^{n} D^{\gamma} v(s)\right\|_{L^{2}} d s \\
& +\Im \int_{0}^{t} \frac{\langle x\rangle^{2 n} D^{\beta} f D^{\beta} \bar{v}}{\langle x\rangle^{n}\left|D^{\beta} v\right|} d s,
\end{aligned}
$$

for all $x \in \mathbb{R}^{N}$. In the case when $|\beta|=\nu+\mu+2 m+1$ with $0 \leq \nu \leq k+1$ and $0 \leq \mu \leq n$, we have

$$
\begin{aligned}
\left\|\langle\cdot\rangle^{n-\mu} D^{\beta} v\right\|_{L^{2}} \leq & \left\|\langle\cdot\rangle^{n-\mu} D^{\beta} v_{0}\right\|_{L^{2}}+(n-\mu) A \int_{0}^{t}\left\|\langle\cdot\rangle^{n-\mu-1} \nabla D^{\beta} v\right\|_{L^{2}} d s \\
& +\int_{0}^{t} \frac{\Im \int_{\mathbb{R}^{N}}\langle x\rangle^{2 n-2 \mu} D^{\beta} f D^{\beta} \bar{v} d x}{\left\|\langle\cdot\rangle^{n-\mu} D^{\beta} v\right\|_{L^{2}}} d s .
\end{aligned}
$$

Proof. We first prove (2.3). Let $|\beta| \leq 2 m$. Applying $\langle x\rangle^{n} D^{\beta}$ to equation (2.1) we obtain

$$
i \partial_{t}\left(\langle x\rangle^{n} D^{\beta} v\right)=-\langle x\rangle^{n} D^{\beta} \Delta v+\langle x\rangle^{n} D^{\beta} f .
$$

Multiplying by $\langle x\rangle^{n} D^{\beta} \bar{v}$ and taking the imaginary part we deduce that

$$
\frac{1}{2} \partial_{t}\left(\left|\langle x\rangle^{n} D^{\beta} v\right|^{2}\right)=-\Im\left(\langle x\rangle^{2 n} D^{\beta} \Delta v D^{\beta} \bar{v}\right)+\Im\left(\langle x\rangle^{2 n} D^{\beta} f D^{\beta} \bar{v}\right) .
$$

Integrating this last equation on $(0, t)$ with $0<t \leq T$, we deduce that

$$
\begin{aligned}
\left|\langle x\rangle^{n} D^{\beta} v\right| \leq & \left|\langle x\rangle^{n} D^{\beta} v_{0}\right| \\
& +\int_{0}^{t}\left|\langle x\rangle^{n} D^{\beta} \Delta v\right| d s+\Im \int_{0}^{t} \frac{\langle x\rangle^{2 n} D^{\beta} f D^{\beta} \bar{v}}{\left|\langle x\rangle^{n} D^{\beta} v\right|} d s .
\end{aligned}
$$


If $|\beta| \leq 2 m-2$, then (2.3) immediately follows from (2.7). Suppose now $2 m-2 \leq$ $|\beta| \leq 2 m$. Since $k-2>\frac{N}{2}$ by (2.2), it follows from Sobolev's embedding theorem that $\left\|\langle\cdot\rangle^{n} D^{\beta} \Delta v(s)\right\|_{L^{\infty}} \leq C\left\|\langle\cdot\rangle^{n} D^{\beta} \Delta v(s)\right\|_{H^{k-2}}$ where $C$ depends on $N$ and $k$. Using Leibniz's formula together with the estimate $\left|D^{\gamma}\langle x\rangle^{n}\right| \leq C(n,|\gamma|)\langle x\rangle^{n}$ (see $[6$, formula (A.3)]), we deduce that

$$
\left\|\langle\cdot\rangle^{n} D^{\beta} \Delta v(s)\right\|_{L^{\infty}} \leq C \sum_{|\gamma| \leq k-2}\left\|\langle\cdot\rangle^{n} D^{\gamma+\beta} \Delta v(s)\right\|_{L^{2}}
$$

for some constant $C$ depending on $N, k, n, m$; hence (2.4) follows from (2.7).

Finally, suppose that $|\beta|=\nu+\mu+2 m+1$ with $0 \leq \nu \leq k+1$ and $0 \leq \mu \leq n$. Applying (2.6) with $n$ replaced with $n-\mu$ and integrating in $x$ we obtain

$$
\begin{aligned}
\frac{1}{2} \frac{d}{d t}\left\|\langle\cdot\rangle^{n-\mu} D^{\beta} v\right\|_{L^{2}}^{2}= & -\Im \int_{\mathbb{R}^{N}}\langle x\rangle^{2 n-2 \mu} \Delta D^{\beta} v D^{\beta} \bar{v} d x \\
& +\Im \int_{\mathbb{R}^{N}}\langle x\rangle^{2 n-2 \mu} D^{\beta} f D^{\beta} \bar{v} d x
\end{aligned}
$$

Integrating by parts the first term in the right-hand side of (2.9), we see that

$$
-\Im \int_{\mathbb{R}^{N}}\langle x\rangle^{2 n-2 \mu} \Delta D^{\beta} v D^{\beta} \bar{v} d x=\Im \int_{\mathbb{R}^{N}} \nabla\langle x\rangle^{2 n-2 \mu} \nabla D^{\beta} v D^{\beta} \bar{v} d x .
$$

If $\mu<n$, we use the estimate $\left|\nabla\langle x\rangle^{2 n-2 \mu}\right| \leq C\langle x\rangle^{2 n-2 \mu-1}$ together with CauchySchwarz to obtain

$$
-\Im \int_{\mathbb{R}^{N}}\langle x\rangle^{2 n-2 \mu} \Delta D^{\beta} v D^{\beta} \bar{v} d x \leq C\left\|\langle\cdot\rangle^{n-\mu-1} \nabla D^{\beta} v\right\|_{L^{2}}\left\|\langle\cdot\rangle^{n-\mu} D^{\beta} v\right\|_{L^{2}} .
$$

If $\mu=n$, then $\nabla\langle x\rangle^{2 n-2 \mu}=0$. In both cases, dividing (2.9) by $\left\|\langle\cdot\rangle^{n-\mu} D^{\beta} v\right\|_{L^{2}}$ and integrating on $(0, t)$, we obtain $(2.5)$.

We now recall the local wellposedness result for $\left(\mathrm{NLS}_{b}\right)$ in the space $\mathcal{X}$ (see $[5$, Theorem 1] and [6, Proposition 4.1]).

Proposition 2.2. Let $\alpha>0$, assume (2.2), $n>\frac{N}{2 \alpha}$, and let $\mathcal{X}$ be defined by (1.10). Let $\lambda \in \mathbb{C}$ and $b \geq 0$. If $v_{0} \in \mathcal{X}$ satisfies (1.11), then there exist $0<T<\frac{1}{b}$ and $a$ unique solution $v \in C([0, T], \mathcal{X})$ of $\left(\mathrm{NLS}_{b}\right)$ satisfying

$$
\inf _{0 \leq t \leq T} \inf _{x \in \mathbb{R}^{N}}\left(\langle x\rangle^{n}|v(t, x)|\right)>0 .
$$

Moreover, $v$ can be extended on a maximal existence interval $\left[0, T_{\max }\right)$ with $0<$ $T_{\max } \leq \frac{1}{b}$ to a solution $v \in C\left(\left[0, T_{\max }\right), \mathcal{X}\right)$ satisfying $(2.10)$ for all $0<T<T_{\max }$. Furthermore, if $T_{\max }<\frac{1}{b}$, then

$$
\|v(t)\|_{\mathcal{X}}+\left(\inf _{x \in \mathbb{R}^{N}}\langle x\rangle^{n}|v(t, x)|\right)^{-1} \underset{t \uparrow T_{\max }}{\longrightarrow} \infty .
$$

In the following section, we will have to estimate $\left|D^{\beta}\left(|v|^{\rho}\right)\right|$ for $\rho \in \mathbb{R}$ and $|\beta| \geq 1$ in terms of $v$ and its derivatives. This is the purpose of the following three lemmas.

Lemma 2.3. Assume (2.2), (1.8), and let $\mathcal{X}$ be defined by (1.10). There exists a constant $C$ such that if $K \geq 1$ and $v \in \mathcal{X}$ satisfies

$$
\|v\|_{\mathcal{X}}+\left(\inf _{x \in \mathbb{R}^{N}}\langle x\rangle^{n}|v(x)|\right)^{-1} \leq K
$$

then

$$
\left\|\frac{D^{\beta} v}{|v|}\right\|_{L^{\infty}} \leq C K^{2}
$$

for all $|\beta| \leq 2 m+2$, where the constant $C$ depends only on $N, k, n, m$. 
Proof. We write

$$
\frac{\left|D^{\beta} v\right|}{|v|}=\frac{\langle x\rangle^{n}\left|D^{\beta} v\right|}{\langle x\rangle^{n}|v|} \leq K\left\|\langle x\rangle^{n} D^{\beta} v\right\|_{L^{\infty}} .
$$

Estimate (2.13) immediately follows if $|\beta| \leq 2 m$. If $2 m+1 \leq|\beta| \leq 2 m+2$, we use the Sobolev embedding (cf. (2.8))

$$
\left\|\langle\cdot\rangle^{n} D^{\beta} v\right\|_{L^{\infty}} \leq C \sum_{|\gamma| \leq k-2}\left\|\langle\cdot\rangle^{n} D^{\gamma+\beta} v\right\|_{L^{2}} \leq C\|v\|_{\mathcal{X}},
$$

since $2 m+1 \leq|\beta+\gamma| \leq 2 m+k=J-n$. This completes the proof.

Lemma 2.4. Given $\rho \in \mathbb{R}$ and $\beta$ a multi-index with $|\beta| \geq 1$, there exists a constant $C$ such that the following estimate holds. If $U \subset \mathbb{R}^{N}$ is an open subset, $v \in$ $C^{|\beta|}(U, \mathbb{C}), v(x) \neq 0$ for all $x \in U$, then $|v|^{\rho} \in C^{|\beta|}(U, \mathbb{R})$ and

$$
\frac{\left|D^{\beta}\left(|v|^{\rho}\right)\right|}{|v|^{\rho}} \leq|\rho| \frac{\left|D^{\beta} v\right|}{|v|}+C \sup _{D} \prod_{\ell=1}^{|\beta|} \frac{\left|D^{\beta_{\ell}} v\right|}{|v|}
$$

where $D$ is the set of $\left(\beta_{\ell}\right)_{1 \leq \ell \leq|\beta|}$ where $\beta_{\ell}$ are multi-indices $0 \leq\left|\beta_{\ell}\right| \leq|\beta|-1$ such that $\sum_{\ell=1}^{|\beta|} \beta_{\ell}=\beta$.

Proof. By the Faà di Bruno's formula (see Corollary 2.10 in $[8]), D^{\beta}\left(\varphi\left(|v|^{2}\right)\right.$ ) is a sum of terms of the form

$$
\varphi^{(\nu)}\left(|v|^{2}\right) \prod_{\ell=1}^{\nu} D^{\gamma_{\ell}}\left(|v|^{2}\right),
$$

with appropriate coefficients, where $\nu \in\{1, \cdots,|\beta|\},\left|\gamma_{\ell}\right| \geq 1$ and $\sum_{\ell=1}^{\nu} \gamma_{\ell}=\beta$. Applying this to $\varphi(s)=s^{\frac{\rho}{2}}$, we see that $D^{\beta}\left(|v|^{\rho}\right)$ is a sum of terms of the form

$$
\boldsymbol{T}=|v|^{\rho-2 \nu} \prod_{\ell=1}^{\nu} D^{\gamma_{\ell}}\left(|v|^{2}\right)
$$

with appropriate coefficients, and the same relations as above on the $\gamma_{\ell}$. For a term $\boldsymbol{T}$ as above, we let $L_{1}$ and $L_{2}$ the (possibly empty) sets of $\ell \in\{1, \cdots, \nu\}$ for which $\left|\gamma_{\ell}\right|=1$ and $\left|\gamma_{\ell}\right| \geq 2$, respectively. If $\nu_{1}=\# L_{1}$ and $\nu_{2}=\# L_{2}$, then clearly $\nu_{1}+\nu_{2}=\nu$, and $\nu_{1}+2 \nu_{2} \leq|\beta|$. Next, we note that $|v|^{2}=v \bar{v}$. Therefore, if $\ell \in L_{1}$, then $\left|D^{\gamma_{\ell}}\left(|v|^{2}\right)\right| \leq 2|v|\left|D^{\gamma_{\ell}} v\right|$; and if $\ell \in L_{2}$, then by Leibniz's rule, $\left|D^{\gamma_{\ell}}\left(|v|^{2}\right)\right|$ is estimated by a sum of terms of the form $\left|D^{\gamma_{\ell}^{1}} v\right|\left|D^{\gamma_{\ell}^{2}} v\right|$ with $\gamma_{\ell}^{1}+\gamma_{\ell}^{2}=\gamma_{\ell}$. Thus we see that $|\boldsymbol{T}|$ is estimated by a sum of terms of the form

$$
|v|^{\rho-2 \nu+\nu_{1}}\left(\prod_{\ell \in L_{1}}\left|D^{\gamma_{\ell}} v\right|\right)\left(\prod_{\ell \in L_{2}}\left|D^{\gamma_{\ell}^{1}} v\right|\left|D^{\gamma_{\ell}^{2}} v\right|\right)
$$

which we rewrite, since $2 \nu=2 \nu_{1}+2 \nu_{2}$, in the form

$$
|v|^{\rho}\left(\prod_{\ell \in L_{1}} \frac{\left|D^{\gamma_{\ell}} v\right|}{|v|}\right)\left(\prod_{\ell \in L_{2}} \frac{\left|D^{\gamma_{\ell}^{1}} v\right|}{|v|} \frac{\left|D^{\gamma_{\ell}^{2}} v\right|}{|v|}\right) .
$$

Therefore, using $\nu_{1}+2 \nu_{2} \leq|\beta|$, we see that $\left|D^{\beta}\left(|v|^{\rho}\right)\right|$ is estimated by a sum of terms of the form

$$
|v|^{\rho} \prod_{\ell=1}^{|\beta|} \frac{\left|D^{\beta_{\ell}} v\right|}{|v|}
$$

with appropriate coefficients, where possibly $\left|\beta_{\ell}\right|=0$ and $\sum_{\ell=1}^{|\beta|} \beta_{\ell}=\beta$. Finally, we notice that the only term where derivatives of order $|\beta|$ appear in the development of $\frac{\left|D^{\beta}\left(|v|^{\rho}\right)\right|}{|v|^{\rho}}$ corresponds to $\nu=1$ and is given by

$$
\frac{\rho}{2}|v|^{-2}\left(v D^{\beta} \bar{v}+\bar{v} D^{\beta} v\right)
$$


which yields the first term in (2.15). Hence (2.15) is proved.

Lemma 2.5. Assume (2.2), (1.8), and let $\mathcal{X}$ be defined by (1.10). Given $\rho>0$, there exists a constant $C$ such that the following inequalities hold. If $K \geq 1$ and $v \in \mathcal{X}$ satisfies (2.12), then

$$
\left\|\frac{D^{\beta}\left(|v|^{\rho}\right)}{|v|^{\rho}}\right\|_{L^{\infty}} \leq C K^{2|\beta|},
$$

and

$$
\left\|\langle\cdot\rangle^{n \rho} D^{\beta}\left(|v|^{\rho}\right)\right\|_{L^{\infty}} \leq C K^{\rho+2|\beta|}
$$

for $|\beta| \leq 2 m+2$. Moreover, if

$$
\eta<n \rho-\frac{N}{2}, \quad \eta \leq-n+M(|\beta|)+n \rho,
$$

then for $C$ possibly larger,

$$
\left\|\langle\cdot\rangle^{\eta} D^{\beta}\left(|v|^{\rho}\right)\right\|_{L^{2}} \leq C K^{\rho+2|\beta|}
$$

for all $|\beta| \leq J$, all $K \geq 1$ and all $v \in \mathcal{X}$ satisfying (2.12), where $\boldsymbol{M}(|\beta|)$ is defined by $(1.9)$.

Proof. Estimates (2.16) and (2.17) are immediate consequences of (2.15) and (2.13).

Suppose now (2.18). In particular $\langle\cdot\rangle^{\eta-n \rho} \in L^{2}\left(\mathbb{R}^{N}\right)$; and so $\left\|\langle\cdot\rangle^{\eta} D^{\beta}\left(|v|^{\rho}\right)\right\|_{L^{2}} \leq$ $C\left\|\langle\cdot\rangle^{n \rho} D^{\beta}\left(|v|^{\rho}\right)\right\|_{L^{\infty}}$. Hence (2.19) in the case $|\beta| \leq 2 m$ follows from (2.17). For $|\beta| \geq 2 m+1$, we argue as follows. By (2.15), $\left|D^{\beta}\left(|v|^{\rho}\right)\right|$ is estimated by a sum of terms of the form

$$
\boldsymbol{T}=|v|^{\rho} \prod_{\ell=1}^{|\beta|} \frac{\left|D^{\beta_{\ell}} v\right|}{|v|}
$$

where $\sum_{\ell=1}^{|\beta|} \beta_{\ell}=\beta$. If all the $\beta_{\ell}$ satisfy $\left|\beta_{\ell}\right| \leq 2 m$, then we can argue as above, and we obtain $\left\|\langle\cdot\rangle^{\eta} \boldsymbol{T}\right\|_{L^{2}} \leq C K^{\rho+2|\beta|}$. Suppose now one of the derivatives in $\boldsymbol{T}$ has order $\geq 2 m+1$, for instance $\left|\beta_{1}\right| \geq 2 m+1$. Then $\left|\beta_{\ell}\right| \leq 2 m$ for all $2 \leq \ell \leq|\beta|$. Indeed, $\sum_{\ell=1}^{|\beta|}\left|\beta_{\ell}\right|=|\beta|$; and for $\ell \geq 2$,

$$
\left|\beta_{\ell}\right| \leq|\beta|-\left|\beta_{1}\right| \leq|\beta|-2 m-1 \leq J-2 m-1 \leq 2 m,
$$

by (1.8) and the last inequality in (2.2). Therefore, we obtain

$$
\boldsymbol{T} \leq K^{\alpha+2|\beta|-1}\langle x\rangle^{-n \rho}\langle x\rangle^{n}\left|D^{\beta_{1}} v\right|,
$$

Using now the second inequality in (2.18), we deduce that

$$
\langle x\rangle^{\eta} \boldsymbol{T} \leq\langle x\rangle^{-n+\boldsymbol{M}(|\beta|)+n \rho} \boldsymbol{T} \leq K^{\alpha+2|\beta|-1}\langle x\rangle^{\boldsymbol{M}(|\beta|)}\left|D^{\beta_{1}} v\right| .
$$

Now $\left|\beta_{1}\right| \leq|\beta|$, so that $\boldsymbol{M}(|\beta|) \leq \boldsymbol{M}\left(\left|\beta_{1}\right|\right)$; hence

$$
\left\|\langle\cdot\rangle^{\eta} \boldsymbol{T}\right\|_{L^{2}} \leq K^{\rho+2|\beta|-1}\left\|\langle\cdot\rangle^{M\left(\left|\beta_{1}\right|\right)} D^{\beta_{1}} v\right\|_{L^{2}} \leq K^{\rho+2|\beta|} .
$$

This completes the proof.

\section{A PRIORI ESTIMATES FOR $\left(\mathrm{NLS}_{b}\right)$}

In this section, we prove a priori estimates for certain solutions of $\left(\mathrm{NLS}_{b}\right)$. These estimates are an essential ingredient in the proof of our main theorem. We assume (1.4) and (1.5)-(1.7). Since $\alpha<\frac{2}{N}$ by (1.4), it follows from the first inequality in (1.6) that

$$
n>\max \left\{\frac{5 N}{2}, \frac{2 N}{\alpha}\right\}
$$


By the second inequality in (3.1), $\frac{N(2-N \alpha)}{n \alpha}<\frac{2-N \alpha}{2}$; by the first inequality in (1.6), $\frac{N(2-N \alpha)}{n \alpha}<\frac{N \alpha}{10}$; by the second inequality in (1.6), $\frac{N(2-N \alpha)}{n \alpha} \leq \frac{1}{k+4}$; and by the last inequality in (1.6), $\frac{N(2-N \alpha)}{n \alpha}<\frac{(N+2) \alpha-2}{2 \alpha(k+2)}$. Therefore, we may fix $\sigma$ satisfying

$$
\frac{N(2-N \alpha)}{n \alpha}<\sigma<\min \left\{\frac{N \alpha}{10}, \frac{2-N \alpha}{2}, \frac{1}{k+4}, \frac{(N+2) \alpha-2}{2 \alpha(k+2)}\right\} .
$$

In particular, it follows from the first inequality in (3.2) that

$$
\frac{n \alpha \sigma}{2-N \alpha}>N
$$

Moreover, it follows from the third inequality in (3.2) that

$$
0<1-\frac{2 \sigma}{2-N \alpha}<1
$$

and from the second inequality in (3.2) that

$$
1-\frac{2-N \alpha}{2}-5 \sigma>0 \text {. }
$$

Also, it follows from the second inequality in (1.7) and (3.3) that

$$
m>\frac{5|\lambda|(1+\alpha|\Im \lambda|)}{|\Im \lambda| \sigma} .
$$

Next, we introduce the following notation. Let

$$
\sigma_{j}= \begin{cases}j \sigma & 0 \leq j \leq 2 m \\ (j+1) \sigma & j=2 m+1, \\ (j+2) \sigma & 2 m+2 \leq j \leq J-2, \\ (j+3) \sigma & j=J-1, \\ (j+4) \sigma & j=J .\end{cases}
$$

In particular, if $j \geq J-2$, then $\sigma_{j} \geq j \sigma \geq 2 m \sigma$. Using (3.6), we deduce that

$$
\frac{10|\lambda|(1+\alpha|\Im \lambda|)}{|\Im \lambda| \sigma_{j}} \leq 1, \quad J-2 \leq j \leq J
$$

Given $\ell \in \mathbb{N}$, we set

$$
\begin{array}{lr}
\|v\|_{1, \ell}=\sup _{0 \leq|\beta| \leq \ell}\left\|\langle\cdot\rangle^{n} D^{\beta} v\right\|_{L^{\infty}}, & \text { if } 0 \leq \ell \leq 2 m, \\
\|v\|_{2, \ell}=\sup _{2 m+1 \leq|\beta| \leq \ell}\left\|\langle\cdot\rangle^{M(|\beta|)} D^{\beta} v\right\|_{L^{2}}, & \text { if } 2 m+1 \leq \ell \leq J,
\end{array}
$$

where $\boldsymbol{M}(\cdot)$ is defined by $(1.9)$. Let $0<T \leq \frac{1}{b}$ and $v \in C([0, T), \mathcal{X})$ satisfy

$$
\inf _{0 \leq s \leq t} \inf _{x \in \mathbb{R}^{N}}\left(\langle x\rangle^{n}|v(s, x)|\right)>0, \quad \text { for all } \quad 0 \leq t<T .
$$

Given $0 \leq t<T$, we define

$$
\begin{aligned}
& \Phi_{1, t}=\sup _{0 \leq s \leq t} \sup _{0 \leq j \leq 2 m}(1-b s)^{\sigma_{j}}\|v(s)\|_{1, j}, \\
& \Phi_{2, t}=\sup _{0 \leq s \leq t} \sup _{2 m+1 \leq j \leq J}(1-b s)^{\sigma_{j}}\|v(s)\|_{2, j}, \\
& \Phi_{3, t}=\sup _{0 \leq s \leq t} \frac{(1-b s)^{\frac{2-N \alpha}{2 \alpha}}}{\inf _{x \in \mathbb{R}^{N}}\langle x\rangle^{n}|v(s, x)|} \\
& \Phi_{4, t}=\sup _{0 \leq s \leq t} \sup _{j \leq 2 m+2}\left((1-b s)^{\sigma_{j}} \sup _{|\beta|=j}\left\|\frac{D^{\beta} v(s)}{|v(s)|}\right\|_{L^{\infty}}\right),
\end{aligned}
$$

and we set

$$
\Phi_{t}=\max \left\{\Phi_{1, t}, \Phi_{2, t}\right\}
$$


and

$$
\Psi_{t}=\max \left\{\Phi_{t}, \Phi_{3, t}, \Phi_{4, t}\right\} .
$$

Note that the norms in the definition of $\Phi_{4, t}$ are finite by (2.13).

Lemma 3.1. Assume (1.4), (1.5)-(1.7) and (3.2), and let $\mathcal{X}$ be defined by (1.10). Let $v_{0} \in \mathcal{X}$ satisfy

$$
\left\|v_{0}\right\|_{\mathcal{X}}+\left(\inf _{x \in \mathbb{R}^{N}}\langle x\rangle^{n}\left|v_{0}(x)\right|\right)^{-1} \leq K,
$$

for some $K \geq 1$, let $0<T \leq \frac{1}{b}$, let $v \in C([0, T), \mathcal{X})$ satisfy $(3.11)$ and $v(0)=v_{0}$. With the notation (3.9)-(3.17), it follows that $\Psi_{t}$ is a continuous function of $t \in$ $[0, T)$, and that

$$
\Psi_{0} \leq K+\Lambda K^{2}
$$

where the constant $\Lambda$ depends only on $N, k, n, m$.

Proof. Since $\|\cdot\|_{j, \ell} \leq\|\cdot\|_{\mathcal{X}}$, it follows that $\Phi_{j, t}$ is a continuous function of $t$ for $j=$ 1,2 and $\Phi_{j, 0} \leq\left\|v_{0}\right\|_{\mathcal{X}} \leq K$. From (3.11) and $v \in C([0, T), \mathcal{X})$, it follows easily that $\Phi_{3, t}$ is also a continuous function of $t$ and that $\Phi_{3,0}=\left(\inf _{x \in \mathbb{R}^{N}}\langle x\rangle^{n}\left|v_{0}(x)\right|\right)^{-1} \leq K$. For $\Phi_{4, t}$ we write

$$
\frac{D^{\beta} v}{|v|}=\frac{\langle x\rangle^{n} D^{\beta} v}{\langle x\rangle^{n}|v|}
$$

We observe that $\left(\langle x\rangle^{n}|v|\right)^{-1}$ is continuous $[0, T) \rightarrow L^{\infty}\left(\mathbb{R}^{N}\right)$ (by (3.11) and $v \in$ $C([0, T], \mathcal{X})$ ), and that $\langle x\rangle^{n} D^{\beta} v$ is continuous $[0, T) \rightarrow L^{\infty}\left(\mathbb{R}^{N}\right)$ (by definition of $\mathcal{X}$ if $|\beta| \leq 2 m$ and by (2.14) if $2 m+1 \leq|\beta| \leq 2 m+2$ ). Hence $\Phi_{4, t}$ is also a continuous function of $t$. Applying (2.13), we see that $\Phi_{4,0} \leq \Lambda K^{2}$ where the constant $\Lambda$ depends only on $N, k, n, m$.

The main result of this section is the following.

Proposition 3.2. Let $\lambda \in \mathbb{C}$ satisfy $\Im \lambda<0$. Assume (1.4), (1.5)-(1.7) and (3.2), and let $\mathcal{X}$ be defined by (1.10). Let $b>0, K>1$, let $v_{0} \in \mathcal{X}$ satisfy (3.18), let $v \in C\left(\left[0, T_{\max }\right), \mathcal{X}\right)$ be the solution of $\left(\mathrm{NLS}_{b}\right)$ given by Proposition 2.2 , and let $\Psi$ be defined by (3.9)-(3.17). Given any $K_{1} \geq K$, there exists $b_{0}>1$ (which depends on $v$ through $K$ and $K_{1}$ only) such that if $b \geq b_{0}$ and

$$
\Psi_{T} \leq K_{1}
$$

for some $0<T<T_{\max }$, then

$$
|v(t, x)| \leq 2\left|v_{0}(x)\right|
$$

and

$$
|v(t, x)|^{\alpha} \leq\left(1+\frac{2-N \alpha}{2 \alpha|\Im \lambda|}\right) \min \left\{2 K^{\alpha}\langle x\rangle^{-n \alpha}, b G(t)\right\}
$$

on $[0, T] \times \mathbb{R}^{N}$, where

$$
G=G(t ; b, \alpha, N)=\frac{(1-b t)^{\frac{2-N \alpha}{2}}}{1-(1-b t)^{\frac{2-N \alpha}{2}}}=\frac{1}{(1-b t)^{-\frac{2-N \alpha}{2}}-1}
$$

Moreover, there is a constant $C_{0}>0$ (which depends on $v$ through $K$ and $K_{1}$ only and is independent of $b$ and $T$ ) such that if $b \geq b_{0}$, then for $0 \leq t \leq T$,

$$
\left\||v(t)|^{\alpha}\right\|_{L^{2}} \leq C_{0} \min \left\{1,(b G(t))^{1-\frac{2 \sigma}{2-N \alpha}}\right\}
$$

and

$$
\left\|\langle\cdot\rangle^{\frac{2 n \alpha \sigma}{2-N \alpha}} D^{\beta}\left(|v(t)|^{\alpha}\right)\right\|_{L^{\infty}} \leq C_{0} \min \left\{1,(b G(t))^{1-\frac{2 \sigma}{2-N \alpha}}\right\}(1-b t)^{-(|\beta|-1) \sigma},
$$


for all $1 \leq|\beta| \leq 2 m$; and

$$
\begin{aligned}
\left\|\langle\cdot\rangle^{M(|\beta|)} v(t) D^{\beta}\left(|v(t)|^{\alpha}\right)\right\|_{L^{2}} \\
\quad \leq C_{0}(1-b t)^{-(|\beta|-1) \sigma} \min \left\{1,(b G(t))^{1-\frac{2 \sigma}{2-N \alpha}}\right\},
\end{aligned}
$$

for all $2 m+1 \leq|\beta| \leq J-2$.

Corollary 3.3. Under the assumptions of Proposition 3.2, it follows that

$$
\begin{aligned}
\left\|\langle\cdot\rangle^{M(|\beta|)} D^{\beta}\left(|v(t)|^{\alpha}\right) D^{\gamma} v(t)\right\|_{L^{2}} \\
\quad \leq C_{0} \min \left\{1,(b G(t))^{1-\frac{2 \sigma}{2-N \alpha}}\right\}(1-b t)^{-(|\beta|+|\gamma|-1) \sigma},
\end{aligned}
$$

for all $0 \leq t \leq T, 2 m+1 \leq|\beta| \leq J-2$ and $0 \leq|\gamma| \leq 2 m+2$.

Proof. We have

$$
\left\|\langle\cdot\rangle^{M(|\beta|)} D^{\beta}\left(|v(t)|^{\alpha}\right) D^{\gamma} v(t)\right\|_{L^{2}} \leq\left\|\langle\cdot\rangle^{M(|\beta|)} D^{\beta}\left(|v(t)|^{\alpha}\right) v(t)\right\|_{L^{2}}\left\|\frac{D^{\gamma} v(t)}{|v(t)|}\right\|_{L^{\infty}} .
$$

Since

$$
\left\|\frac{D^{\gamma} v(t)}{|v(t)|}\right\|_{L^{\infty}} \leq K_{1}(1-b t)^{-|\gamma| \sigma},
$$

by (3.20) and (3.15), estimate (3.27) follows by applying (3.26).

Proof of Proposition 3.2. In the estimates that follow, we denote by $C_{0}>0$ a constant depending possibly on $\beta, \alpha, N, K, K_{1}, \lambda$, etc, but not on $b, v, T$ and $T_{\max }$, whose exact value is irrelevant and can change from line to line. We consider $b \geq 1$ and we proceed in several steps.

Step 1. Proof of (3.22). From equation $\left(\mathrm{NLS}_{b}\right)$ it follows that

$$
\partial_{t}|v|=L+\Im \lambda(1-b t)^{-\frac{4-N \alpha}{2}}|v|^{\alpha+1},
$$

where

$$
L(t, x)=-\frac{\Im(\bar{v}(t, x) \Delta v(t, x))}{|v(t, x)|} .
$$

Multiplying both sides of (3.28) by $|v|^{-\alpha-1}$ we see that

$$
-\frac{1}{\alpha} \frac{\partial}{\partial t}|v|^{-\alpha}=|v|^{-\alpha-1} L+\Im \lambda(1-b t)^{-\frac{4-N \alpha}{2}} .
$$

Let $0<t \leq T$. Integrating (3.30) in $t$ we obtain

$$
\begin{aligned}
\frac{1}{|v(t, x)|^{\alpha}=} & \frac{1}{\left|v_{0}(x)\right|^{\alpha}}+\frac{2 \alpha|\Im \lambda|}{b(2-N \alpha)}\left[(1-b t)^{-\frac{2-N \alpha}{2}}-1\right] \\
& -\alpha \int_{0}^{t}|v(s, x)|^{-\alpha-1} L(s, x) d s .
\end{aligned}
$$

it follows that

$$
|v(t, x)|^{\alpha}=\frac{\left|v_{0}(x)\right|^{\alpha}}{H(t, x)},
$$

where

with

$$
H(t, x)=1+f(t, x)+\frac{2 \alpha|\Im \lambda|}{b G(t)(2-N \alpha)}\left|v_{0}(x)\right|^{\alpha}
$$

$$
f(t, x)=-\alpha\left|v_{0}(x)\right|^{\alpha} \int_{0}^{t}|v(s, x)|^{-\alpha-1} L(s, x) d s .
$$

By (3.20) and (3.14) we have

$$
\frac{1}{\langle x\rangle^{\alpha n}|v(t, x)|^{\alpha}} \leq K_{1}^{\alpha}(1-b t)^{-\frac{2-N \alpha}{2}}
$$


and by (3.20), (3.15) and (3.7),

$$
\frac{|\Delta v(t, x)|}{|v(t, x)|} \leq K_{1}(1-b t)^{-2 \sigma}
$$

Using (3.18), (3.35), (3.29) and (3.36) we obtain

$$
\begin{aligned}
\left|v_{0}(x)\right|^{\alpha}|v(s, x)|^{-\alpha-1}|L(s, x)| & =\left(\langle x\rangle^{n}\left|v_{0}\right|\right)^{\alpha}\left(\langle x\rangle^{n}|v(s, x)|\right)^{-\alpha} \frac{|L(s, x)|}{|v(s, x)|} \\
& \leq K^{\alpha} K_{1}^{\alpha+1}(1-b s)^{-\frac{2-N \alpha}{2}-2 \sigma} \\
& \leq K_{1}^{2 \alpha+1}(1-b s)^{-\frac{2-N \alpha}{2}-2 \sigma},
\end{aligned}
$$

on $[0, T] \times \mathbb{R}^{N}$. Note that by the second inequality in (3.2),

$$
\frac{2-N \alpha}{2}+2 \sigma<1
$$

so that

$$
|f(t, x)| \leq \frac{\alpha K_{1}^{2 \alpha+1}}{b\left(1-\frac{2-N \alpha}{2}-2 \sigma\right)} .
$$

We choose $b_{0} \geq 1$ sufficiently large so that

$$
\frac{\alpha K_{1}^{2 \alpha+1}}{b_{0}\left(1-\frac{2-N \alpha}{2}-2 \sigma\right)} \leq \min \left\{\frac{1}{4}, \frac{2^{\alpha}-1}{2^{\alpha}+1}\right\}
$$

and we deduce that if $b \geq b_{0}$, then

$$
|f(t, x)| \leq \min \left\{\frac{1}{4}, \frac{2^{\alpha}-1}{2^{\alpha}+1}\right\}
$$

on $[0, T] \times \mathbb{R}^{N}$. In particular, $1+f(t, x) \geq \frac{1}{2}$, so that

$$
\begin{aligned}
\frac{1}{H(t, x)} & \leq \frac{2}{1+\frac{4 \alpha|\Im \lambda|}{b G(t)(2-N \alpha)}\left|v_{0}(x)\right|^{\alpha}} \\
& \leq \min \left\{2, \frac{2-N \alpha}{2 \alpha|\Im \lambda|\left|v_{0}(x)\right|^{\alpha}} b G(t)\right\} .
\end{aligned}
$$

Applying (3.32), (3.42) and using (3.18) we obtain

$$
|v(t, x)|^{\alpha} \leq \min \left\{2 K^{\alpha}\langle x\rangle^{-n \alpha}, \frac{2-N \alpha}{2 \alpha|\Im \lambda|} b G(t)\right\},
$$

from which estimate (3.22) follows.

STEP 2. Proof of (3.24). Since $\langle x\rangle^{-n \alpha} \in L^{2}\left(\mathbb{R}^{N}\right)$ by the second inequality in (3.1), it follows from the first inequality in (3.22) that $\left\||v|^{\alpha}\right\|_{L^{2}} \leq C_{0}$. Moreover, it follows from (3.22) and $\frac{2 \sigma}{2-N \alpha} \geq \frac{N}{2 n \alpha}$ (by (3.3)) that if $b G(t) \leq 1$, then

$$
\begin{aligned}
\left\||v|^{\alpha}\right\|_{L^{2}} & \leq C_{0}\left(\left\|\langle\cdot\rangle^{-n \alpha}\right\|_{L^{2}\left(\langle x\rangle>(b G(t))^{-\frac{1}{n \alpha}}\right)}+\|b G\|_{L^{2}\left(\langle x\rangle<(b G(t))^{-\frac{1}{n \alpha}}\right)}\right) \\
& \leq C_{0}(b G(t))^{1-\frac{N}{2 n \alpha}} \leq C_{0}(b G(t))^{1-\frac{2 \sigma}{2-N \alpha}}
\end{aligned}
$$

hence (3.24) is proved.

SteP 3. Further estimates of $v$ and $H$ and proof of (3.21). For any $x \in \mathbb{R}^{N}$ and $0 \leq s \leq t \leq T$, it follows from (3.32) and $G(t) \leq G(s)$ that

$$
\left|\frac{v(t, x)}{v(s, x)}\right|^{\alpha}=\frac{1+f(s, x)+\frac{2 \alpha|\Im \lambda|\left|v_{0}\right|^{\alpha}}{(2-N \alpha) b G(s)}}{1+f(t, x)+\frac{2 \alpha|\Im \lambda|\left|v_{0}\right|^{\alpha}}{(2-N \alpha) b G(t)}} \leq \frac{1+f(s, x)+\frac{2 \alpha|\Im \lambda|\left|v_{0}\right|^{\alpha}}{(2-N \alpha) b G(s)}}{1+f(t, x)+\frac{2 \alpha|\Im \lambda|\left|v_{0}\right|^{\alpha}}{(2-N \alpha) b G(s)}}
$$

Using (3.41), we deduce that

$$
\left|\frac{v(t, x)}{v(s, x)}\right|^{\alpha} \leq \min \left\{2,2^{\alpha}\right\} .
$$


This proves (3.21). Moreover, if $t \geq 0$ and $0 \leq \nu \leq 1$, then $\min \{1, t\} \leq \min \left\{1, t^{\nu}\right\}$. Thus it follows from (3.42) and (3.4) that

$$
\frac{1}{H(t, x)} \leq C \min \left\{1,\left(\frac{b G(t)}{\left|v_{0}\right|^{\alpha}}\right)^{1-\frac{2 \sigma}{2-N \alpha}}\right\} \text {. }
$$

Since

it follows that

$$
\left(\frac{b G(t)}{\left|v_{0}\right|^{\alpha}}\right)^{1-\frac{2 \sigma}{2-N \alpha}} \leq K^{\alpha}\langle x\rangle^{n \alpha\left(1-\frac{2 \sigma}{2-N \alpha}\right)}(b G(t))^{1-\frac{2 \sigma}{2-N \alpha}}
$$

$$
\frac{1}{H(t, x)} \leq C K^{\alpha}\langle x\rangle^{n \alpha\left(1-\frac{2 \sigma}{2-N \alpha}\right)} \min \left\{1,(b G(t))^{1-\frac{2 \sigma}{2-N \alpha}}\right\} .
$$

STEP 4. We prove that if $|\gamma| \leq 2 m+2$ then

$$
\left\|\langle\cdot\rangle^{\frac{2 n \alpha \sigma}{2-N \alpha}} \frac{D^{\gamma}\left(\left|v_{0}\right|^{\alpha}\right)}{H(t, \cdot)}\right\|_{L^{\infty}} \leq C_{0} \min \left\{1,(b G(t))^{1-\frac{2 \sigma}{2-N \alpha}}\right\}
$$

for all $0 \leq t \leq T$, and if $2 m+1 \leq|\gamma| \leq J-2$, then

$$
\left\|\langle\cdot\rangle^{M(|\gamma|)} v(t) \frac{D^{\gamma}\left(\left|v_{0}\right|^{\alpha}\right)}{H(t, \cdot)}\right\|_{L^{2}} \leq C_{0} \min \left\{1,(b G(t))^{1-\frac{2 \sigma}{2-N \alpha}}\right\},
$$

for all $0 \leq t \leq T$. Indeed, suppose first $|\gamma| \leq 2 m+2$. It follows from (2.17) that

$$
\left|D^{\gamma}\left(\left|v_{0}\right|^{\alpha}\right)\right| \leq C\langle x\rangle^{-\alpha n} K^{\alpha+2|\gamma|} .
$$

Estimates (3.48) and (3.45) yield (3.46). Suppose now $|\gamma| \geq 2 m+1$. We have

$$
\left\|\langle\cdot\rangle^{M(|\gamma|)} v(t) \frac{D^{\gamma}\left(\left|v_{0}\right|^{\alpha}\right)}{H(t, \cdot)}\right\|_{L^{2}} \leq\left\|\langle\cdot\rangle^{n} v(t)\right\|_{L^{\infty}}\left\|\langle\cdot\rangle^{-n+M(|\gamma|)} \frac{D^{\gamma}\left(\left|v_{0}\right|^{\alpha}\right)}{H(t, \cdot)}\right\|_{L^{2}} .
$$

Using (3.45), we see that

$$
\begin{aligned}
\|\langle\cdot\rangle^{-n+M(|\gamma|)} & \frac{D^{\gamma}\left(\left|v_{0}\right|^{\alpha}\right)}{H(t, \cdot)} \|_{L^{2}} \\
& \leq C K^{\alpha} \min \left\{1,(b G(t))^{1-\frac{2 \sigma}{2-N \alpha}}\right\}\left\|\langle\cdot\rangle^{\eta} D^{\gamma}\left(\left|v_{0}\right|^{\alpha}\right)\right\|_{L^{2}},
\end{aligned}
$$

where

$$
\eta=-n+M(|\gamma|)+n \alpha\left(1-\frac{2 \sigma}{2-N \alpha}\right)
$$

Applying (3.3), we see that

$$
\eta \leq n \alpha\left(1-\frac{2 \sigma}{2-N \alpha}\right)<n \alpha-\frac{N}{2}
$$

Moreover,

$$
\eta<-n+M(|\gamma|)+n \alpha
$$

Therefore, we deduce from (2.19) that $\left\|\langle\cdot\rangle^{\eta} D^{\gamma}\left(|v|^{\alpha}\right)\right\|_{L^{2}} \leq C K^{\alpha+2|\gamma|}$. Applying (3.49) and (3.50), we conclude that (3.47) holds.

SteP 5. Development of $D^{\beta}\left(|v|^{\alpha}\right)$. We differentiate (3.32). Given $1 \leq|\beta| \leq$ $J-2$, the development of $D^{\beta}\left(|v|^{\alpha}\right)$ contains the term

$$
\boldsymbol{A}_{1}=\frac{D^{\beta}\left(\left|v_{0}(x)\right|^{\alpha}\right)}{H(t, x)}
$$

and terms of the form

$$
\boldsymbol{A}_{2}=\frac{D^{\rho}\left(\left|v_{0}(x)\right|^{\alpha}\right)}{H(t, x)} \prod_{j=1}^{p} \frac{D^{\gamma_{j}} H(t, x)}{H(t, x)}
$$


where

$$
\gamma+\rho=\beta, \quad 1 \leq p \leq|\gamma|, \quad\left|\gamma_{j}\right| \geq 1, \quad \sum_{j=1}^{p} \gamma_{j}=\gamma .
$$

The term $\boldsymbol{A}_{1}$ is estimated by (3.46) and (3.47). Since $(1-b t)^{-(|\beta|-1) \sigma} \geq 1$, the contribution of $\boldsymbol{A}_{1}$ satisfies estimates (3.25) and (3.26).

It remains to estimate the terms of the form $\boldsymbol{A}_{2}$. In view of (3.33),

$$
\begin{aligned}
\frac{D^{\gamma_{j}} H(t, x)}{H(t, x)} & =\frac{2 \alpha|\Im \lambda|}{b G(t)(2-N \alpha)} \frac{D^{\gamma_{j}}\left(\left|v_{0}(x)\right|^{\alpha}\right)}{H(t, x)}+\frac{D^{\gamma_{j}} f(t, x)}{H(t, x)} \\
& =: \boldsymbol{B}_{1}^{j}+\boldsymbol{B}_{2}^{j} .
\end{aligned}
$$

STEP 6. Estimates of $\boldsymbol{B}_{1}^{j}$. Using (3.42), we see that

$$
\left|\boldsymbol{B}_{1}^{j}\right| \leq \frac{\left|D^{\gamma_{j}}\left(\left|v_{0}(x)\right|^{\alpha}\right)\right|}{\left|v_{0}(x)\right|^{\alpha}}
$$

Applying (2.16) with $\rho=\alpha$, we obtain

$$
\left\|\boldsymbol{B}_{1}^{j}\right\|_{L \infty} \leq C_{0}
$$

for $\left|\gamma_{j}\right| \leq 2 m+2$.

STEP 7. Estimates of $\boldsymbol{B}_{2}^{j}$. Using (3.34) and (3.29), we write

$$
f(t, x)=\alpha \int_{0}^{t}|v(s, x)|^{-\alpha-2}\left|v_{0}(x)\right|^{\alpha} \Im(\bar{v}(s, x) \Delta v(s, x)) d s,
$$

and we see that the development of $D^{\gamma_{j}} f(t, x)$ is estimated by terms of the form

$$
\int_{0}^{t}\left|D^{\gamma_{j 1}}\left(\left|v_{0}\right|^{\alpha}\right)\right|\left|D^{\gamma_{j 2}} \Delta v\right|\left|D^{\gamma_{j 3}} v\right|\left|D^{\gamma_{j 4}}\left(|v|^{-\alpha-2}\right)\right|,
$$

where $\gamma_{j 1}+\gamma_{j 2}+\gamma_{j 3}+\gamma_{j 4}=\gamma_{j}$. Using (2.15) with $\beta$ replaced by $\gamma_{j 3}$ and $\rho$ replaced by $-\alpha-2$, we are led to estimate terms of the form

$$
\int_{0}^{t}|v|^{-\alpha}\left|D^{\gamma_{j 1}}\left(\left|v_{0}\right|^{\alpha}\right)\right| \frac{\left|D^{\gamma_{j 2}} \Delta v\right|}{|v|} \frac{\left|D^{\gamma_{j 3}} v\right|}{|v|} \prod_{\ell=1}^{\left|\gamma_{j 4}\right|} \frac{\left|D^{\beta_{\ell}} v\right|}{|v|}
$$

where $\sum_{\ell=1}^{\left|\gamma_{j 4}\right|} \beta_{\ell}=\gamma_{j 4}$. Note that we can incorporate the term $\frac{\left|D^{\gamma_{j 3}} v\right|}{|v|}$ into the product. Moreover, $\frac{\left|D^{\beta_{\ell}} v\right|}{|v|}=1$ if $\left|\beta_{\ell}\right|=0$, so we can incorporate as many such terms as we like into the product. Thus, we need only estimate terms of the form

$$
\boldsymbol{D}=\frac{1}{H(t, x)} \int_{0}^{t}|v|^{-\alpha}\left|D^{\gamma_{j 1}}\left(\left|v_{0}\right|^{\alpha}\right)\right| \frac{\left|D^{\gamma_{j 2}} \Delta v\right|}{|v|} \prod_{\ell=1}^{\left|\gamma_{j 3}\right|} \frac{\left|D^{\beta_{\ell}} v\right|}{|v|}
$$

where

$$
\gamma_{j 1}+\gamma_{j 2}+\gamma_{j 3}=\gamma_{j}, \quad \sum_{\ell=1}^{\left|\gamma_{j 3}\right|} \beta_{\ell}=\gamma_{j 3} .
$$

To estimate the terms $\boldsymbol{D}$, we consider separately two cases. If a term $\boldsymbol{D}$ contains only derivatives $\left|\gamma_{j k}\right| \leq 2 m$ for $k=1,2$ and $\left|\beta_{\ell}\right| \leq 2 m$ for $\ell \leq\left|\gamma_{j 3}\right|$, then we call such a term $\boldsymbol{D}^{\text {low }}$ and we estimate its $L^{\infty}$ norm. If a term $\boldsymbol{D}$ contains a derivative of order $\left|\gamma_{j k}\right| \geq 2 m+1$ or $\left|\beta_{\ell}\right| \geq 2 m+1$, then we call such a term $\boldsymbol{D}^{\text {high }}$ and we estimate its $L^{2}$ norm.

Recall that $\frac{1}{H} \leq 2$ by (3.42). Writing

$$
|v|^{-\alpha}\left|D^{\gamma_{j 1}}\left(\left|v_{0}\right|^{\alpha}\right)\right|=\left(\langle x\rangle^{n}|v|\right)^{-\alpha}\left|\langle x\rangle^{n \alpha} D^{\gamma_{j 1}}\left(\left|v_{0}\right|^{\alpha}\right)\right|,
$$


we deduce from (2.17), (3.20), (3.14), (3.15), and (3.7) (recall that $\left|\gamma_{j 2}\right|+2 \leq 2 m+2$ ) that

$$
\begin{aligned}
\left\|\boldsymbol{D}^{\mathrm{low}}\right\|_{L^{\infty}} & \leq C_{0} \int_{0}^{t}(1-b s)^{-\frac{2-N \alpha}{2}-\sigma\left(\left|\gamma_{j 2}\right|+4\right)-\sigma \sum_{\ell=1}^{\left|\gamma_{j 3}\right|}\left|\beta_{\ell}\right|} d s \\
& =C_{0} \int_{0}^{t}(1-b s)^{-\frac{2-N \alpha}{2}-\left(\left|\gamma_{j 2}\right|+\left|\gamma_{j 3}\right|+4\right) \sigma} d s \\
& \leq C_{0} \int_{0}^{t}(1-b s)^{-\frac{2-N \alpha}{2}-\left(\left|\gamma_{j}\right|+4\right) \sigma} d s \\
& =C_{0} \int_{0}^{t}(1-b s)^{-\frac{2-N \alpha}{2}-5 \sigma-\left(\left|\gamma_{j}\right|-1\right) \sigma} d s .
\end{aligned}
$$

Since $\left|\gamma_{j}\right|-1 \geq 0$ (by (3.53)) and $-\frac{2-N \alpha}{2}-5 \sigma>-1$ (by (3.5)), we obtain

$$
\begin{aligned}
\left\|\boldsymbol{D}^{\text {low }}\right\|_{L^{\infty}} & \leq C_{0}(1-b t)^{-\left(\left|\gamma_{j}\right|-1\right) \sigma} \int_{0}^{t}(1-b s)^{-\frac{2-N \alpha}{2}-5 \sigma} d s \\
& \leq \frac{C_{0}}{b}(1-b t)^{-\left(\left|\gamma_{j}\right|-1\right) \sigma} .
\end{aligned}
$$

Suppose now that $\max \left\{\left|\gamma_{j 1}\right|,\left|\gamma_{j 2}\right|,\left|\beta_{\ell}\right|\right\} \geq 2 m+1$. Then, all the other derivatives are of order $\leq 2 m$, see the proof of Lemma 2.5. We consider two cases. If $\left|\gamma_{j 1}\right| \geq$ $2 m+1$, then we rename $\boldsymbol{D}^{\text {high }}$ as $\boldsymbol{D}_{1}^{\text {high }}$. If $\left|\gamma_{j 2}\right| \geq 2 m+1$ or if one of the $\beta_{\ell}$ has order $\geq 2 m+1$, then we rename $\boldsymbol{D}^{\text {high }}$ as $\boldsymbol{D}_{2}^{\text {high }}$. If $\left|\gamma_{j 1}\right| \geq 2 m+1$, then it follows from (3.47) that

$$
\left\|\langle\cdot\rangle^{M\left(\left|\gamma_{j 1}\right|\right)} v(t) \frac{D^{\gamma_{j 1}}\left(\left|v_{0}\right|^{\alpha}\right)}{H(t, \cdot)}\right\|_{L^{2}} \leq C_{0} \min \left\{1,(b G(t))^{1-\frac{2 \sigma}{2-N \alpha}}\right\},
$$

hence using (3.20), (3.14), (3.15), (3.7), and $\boldsymbol{M}\left(\left|\gamma_{1}\right|\right) \geq \boldsymbol{M}(|\beta|)$,

$$
\begin{aligned}
& \left\|\langle\cdot\rangle^{-n \alpha+M(|\beta|)} v(t) \boldsymbol{D}_{1}^{\mathrm{high}}\right\|_{L^{2}} \\
& \leq C_{0} \min \left\{1,(b G(t))^{1-\frac{2 \sigma}{2-N \alpha}}\right\} \int_{0}^{t}(1-b s)^{-\frac{2-N \alpha}{2}-\left(\left|\gamma_{j 2}\right|+\left|\gamma_{j 3}\right|+4\right) \sigma} \\
& \leq C_{0} \min \left\{1,(b G(t))^{1-\frac{2 \sigma}{2-N \alpha}}\right\} \frac{(1-b t)^{-\left(\left|\gamma_{j}\right|-1\right) \sigma}}{b},
\end{aligned}
$$

as in (3.59). To estimate $\boldsymbol{D}_{2}^{\text {high }}$, we suppose for instance $\left|\gamma_{j 2}\right| \geq 2 m+1$ (the case where one of the $\beta_{\ell}$ has order $\geq 2 m+1$ is treated similarly). We use again $\frac{1}{H} \leq 2$, and we observe that by $(3.44)$,

$$
|v(t)| \boldsymbol{D}_{2}^{\text {high }} \leq 2^{1+\frac{1}{\alpha}} \int_{0}^{t}\left(\langle x\rangle^{n}|v|\right)^{-\alpha}\left|\langle x\rangle^{\alpha n} D^{\gamma_{j 1}}\left(\left|v_{0}\right|^{\alpha}\right)\right|\left|D^{\gamma_{j 2}} \Delta v\right| \prod_{\ell=1}^{\left|\gamma_{j 3}\right|} \frac{\left|D^{\beta_{\ell}} v\right|}{|v|} .
$$

Note also that

$$
\left\|\langle\cdot\rangle^{M(|\beta|+2)} D^{\gamma_{j 2}} \Delta v\right\|_{L^{2}} \leq\left\|\langle\cdot\rangle^{M\left(\left|\gamma_{j 2}\right|+2\right)} D^{\gamma_{j 2}} \Delta v\right\|_{L^{2}} \leq K_{1}(1-b s)^{\sigma\left(\left|\gamma_{j 2}\right|+4\right)}
$$

by (3.20), (3.13), and (3.7). Using (3.20), (3.14), (3.15), and (3.7), and since $\boldsymbol{M}(|\beta|+2) \geq \boldsymbol{M}(|\beta|)-2$ we obtain

$$
\begin{aligned}
\left\|\langle\cdot\rangle^{M(|\beta|)-2} v(t) \boldsymbol{D}_{2}^{\text {high }}\right\|_{L^{2}} & \leq C_{0} \int_{0}^{t}(1-b s)^{-\frac{2-N \alpha}{2}-\left(\left|\gamma_{j 2}\right|+\left|\gamma_{j 3}\right|+4\right) \sigma} \\
& \leq \frac{C_{0}}{b}(1-b t)^{-\left(\left|\gamma_{j}\right|-1\right) \sigma}
\end{aligned}
$$

as in (3.59).

STEP 8. Proof of (3.25). As observed above (Step 5), we need only show that the terms of the form $\boldsymbol{A}_{2}$ (given by (3.52)) satisfy (3.25). Since $|\beta| \leq 2 m$, all the 
derivatives $\rho$ and $\gamma_{j}$ also have order $\leq 2 m$. We use (3.46) for the term $\frac{D^{\rho}\left(\left|v_{0}(x)\right|^{\alpha}\right)}{H(t, x)}$. For the terms $\frac{D^{\gamma_{j} H(t, x)}}{H(t, x)}$, we use the decomposition (3.54). We have $\left\|\boldsymbol{B}_{1}^{j}\right\|_{L^{\infty}} \leq C_{0}$ by (3.55). Moreover, $\frac{1}{H(t, x)} \leq 2$ by (3.42), so that estimate (3.59) yields

$$
\left\|\boldsymbol{B}_{2}^{j}\right\|_{L^{\infty}} \leq 2\left\|\boldsymbol{D}^{\mathrm{low}}\right\|_{L^{\infty}} \leq \frac{C_{0}}{b}(1-b t)^{-\left(\left|\gamma_{j}\right|-1\right) \sigma} \leq C_{0}(1-b t)^{-\left(\left|\gamma_{j}\right|-1\right) \sigma} .
$$

Therefore, given $p_{0} \in\{1, \cdots, p\}$, we obtain

$$
\left\|\prod_{j=p_{0}}^{p} \frac{D^{\gamma_{j}} H(t, x)}{H(t, x)}\right\|_{L^{\infty}} \leq C_{0} \prod_{j=p_{0}}^{p}(1-b t)^{-\left(\left|\gamma_{j}\right|-1\right) \sigma} \leq C_{0}(1-b t)^{-\sum_{p_{0}}^{p}\left(\left|\gamma_{j}\right|-1\right) \sigma}
$$

and so,

$$
\left\|\prod_{j=p_{0}}^{p} \frac{D^{\gamma_{j}} H(t, x)}{H(t, x)}\right\|_{L^{\infty}} \leq C_{0}(1-b t)^{-\sigma\left(\sum_{p_{0}}^{p}\left(\left|\gamma_{j}\right|\right)+\sigma\right.} .
$$

Therefore, using (3.46) for the term $\frac{D^{\rho}\left(\left|v_{0}(x)\right|^{\alpha}\right)}{H(t, x)}$ and (3.62) with $p_{0}=1$,

$$
\left\|\langle\cdot\rangle^{\frac{2 n \alpha \sigma}{2-N \alpha}} \boldsymbol{A}_{2}\right\|_{L^{\infty}} \leq C_{0} \min \left\{1,(b G(t))^{1-\frac{2 \sigma}{2-N \alpha}}\right\}(1-b t)^{-\sigma\left(\sum_{1}^{p}\left(\left|\gamma_{j}\right|\right)+\sigma\right.} .
$$

since $\sum_{1}^{p}\left|\gamma_{j}\right|=|\gamma| \leq|\beta|$. This proves estimate (3.25).

SteP 9. Proof of (3.26). As observed above (Step 5), we need only show that the terms of the form $\boldsymbol{A}_{2}$ (given by (3.52)) satisfy (3.26). Let $2 m+1 \leq|\beta| \leq J-2$.

If all the derivatives $\rho$ and $\gamma_{j}$ have order $\leq 2 m$, then estimate (3.63) holds. Now using (3.20) and (3.12), $\left\|\langle\cdot\rangle^{n} v(t)\right\|_{L^{\infty}} \leq K_{1}$, so that

$$
\begin{aligned}
\left\|\langle\cdot\rangle^{\boldsymbol{M}(|\beta|)} v(t) \boldsymbol{A}_{2}\right\|_{L^{2}} & \leq C_{0}\left\|\langle\cdot\rangle^{-n+\boldsymbol{M}(|\beta|)} \boldsymbol{A}_{2}\right\|_{L^{2}} \leq C_{0}\left\|\boldsymbol{A}_{2}\right\|_{L^{2}} \\
& \leq C_{0}\left\|\langle\cdot\rangle^{\frac{2 n \alpha \sigma}{2-N \alpha}} \boldsymbol{A}_{2}\right\|_{L^{\infty}}
\end{aligned}
$$

by (3.3). Thus we see that $\boldsymbol{A}_{2}$ satisfies (3.26).

Suppose now $|\rho| \geq 2 m+1$. It follows that $\left|\gamma_{j}\right| \leq 2 m$ for all $j$, see the proof of Lemma 2.5. In particular, estimate (3.62) holds. Using (3.47) for the term $\frac{D^{\rho}\left(\left|v_{0}(x)\right|^{\alpha}\right)}{H(t, x)}$, we see that $\boldsymbol{A}_{2}$ also satisfies (3.26) in this case.

Suppose next that one of the $\gamma_{j}$ 's have order $\geq 2 m+1$, for instance $\left|\gamma_{1}\right| \geq 2 m+1$. Assume also that $p \geq 2$, the case $p=1$ being simpler. It follows (see the proof of Lemma 2.5) that $|\rho| \leq 2 m$ and $\left|\gamma_{j}\right| \leq 2 m$ for $j \geq 2$. Using (3.54), we deduce that

$$
\left|\frac{D^{\gamma_{1}} H(t, x)}{H(t, x)}\right| \leq C_{0} \frac{\left|D^{\gamma_{1}}\left(\left|v_{0}(x)\right|^{\alpha}\right)\right|}{b G(t) H(t, x)}+\frac{\left|D^{\gamma_{1}} f(t, x)\right|}{H(t, x)} .
$$

Writing

$$
\boldsymbol{A}_{2}=\frac{D^{\rho}\left(\left|v_{0}(x)\right|^{\alpha}\right)}{H(t, x)} \frac{D^{\gamma_{1}} H(t, x)}{H(t, x)} \prod_{j=2}^{p} \frac{D^{\gamma_{j}} H(t, x)}{H(t, x)},
$$

we are led to estimate

$$
\boldsymbol{E}_{1}=\frac{1}{b G(t)} \frac{D^{\rho}\left(\left|v_{0}(x)\right|^{\alpha}\right)}{H(t, x)} \frac{D^{\gamma_{1}}\left(\left|v_{0}(x)\right|^{\alpha}\right)}{H(t, x)} \prod_{j=2}^{p} \frac{D^{\gamma_{j}} H(t, x)}{H(t, x)}
$$

and

$$
\boldsymbol{E}_{2}=\frac{D^{\rho}\left(\left|v_{0}(x)\right|^{\alpha}\right)}{H(t, x)} \frac{D^{\gamma_{1}} f(t, x)}{H(t, x)} \prod_{j=2}^{p} \frac{D^{\gamma_{j}} H(t, x)}{H(t, x)}
$$

Since

$$
\frac{1}{b G(t) H(t, x)} \leq \frac{C_{0}}{\left|v_{0}(x)\right|^{\alpha}}
$$


by (3.42), we deduce that

$$
\left|\boldsymbol{E}_{1}\right| \leq C_{0} \frac{\left|D^{\rho}\left(\left|v_{0}(x)\right|^{\alpha}\right)\right|}{\left|v_{0}(x)\right|^{\alpha}} \frac{\left|D^{\gamma_{1}}\left(\left|v_{0}(x)\right|^{\alpha}\right)\right|}{H(t, x)} \prod_{j=2}^{p} \frac{\left|D^{\gamma_{j}} H(t, x)\right|}{H(t, x)}
$$

We use (2.16) for the first term, (3.47) for the second term, and (3.62) with $p_{0}=2$ for the product, and we obtain

$$
\left\|\langle\cdot\rangle^{\boldsymbol{M}\left(\left|\gamma_{1}\right|\right)} v(t) \boldsymbol{E}_{1}\right\|_{L^{2}} \leq C_{0}(1-b t)^{-(|\beta|-1) \sigma} \min \left\{1,(b G(t))^{1-\frac{2 \sigma}{2-N \alpha}}\right\},
$$

so that $\boldsymbol{E}_{1}$ satisfies (3.26). (Recall that $\boldsymbol{M}\left(\left|\gamma_{1}\right|\right) \geq \boldsymbol{M}(|\beta|)$.)

We now estimate $\boldsymbol{E}_{2}$. With the notation (3.56) (with $j=1$ ) we are led to estimate

$$
\boldsymbol{F}=\frac{D^{\rho}\left(\left|v_{0}(x)\right|^{\alpha}\right)}{H(t, x)} \boldsymbol{D} \prod_{j=2}^{p} \frac{D^{\gamma_{j}} H(t, x)}{H(t, x)},
$$

with $\boldsymbol{D}=\boldsymbol{D}^{\text {low }}, \boldsymbol{D}_{1}^{\text {high }}$ or $\boldsymbol{D}_{2}^{\text {high }}$. In the cases $\boldsymbol{D}=\boldsymbol{D}^{\text {low }}$ and $\boldsymbol{D}=\boldsymbol{D}_{2}^{\text {high }}$, we use (3.46) for the first term and (3.62) for the product, and we obtain (since $\left.\sum_{2}^{p}\left|\gamma_{j}\right|=|\gamma|-\left|\gamma_{1}\right| \leq|\beta|-\left|\gamma_{1}\right|\right)$

$$
\begin{aligned}
\left\|\langle\cdot\rangle^{M(|\beta|)} v(t) \boldsymbol{F}\right\|_{L^{2}} \leq & C_{0} \min \left\{1,(b G(t))^{1-\frac{2 \sigma}{2-N \alpha}}\right\} \\
& \times(1-b t)^{-\left(|\beta|-\left|\gamma_{1}\right|-1\right) \sigma}\left\|\langle\cdot\rangle^{\boldsymbol{M}(|\beta|)-\frac{2 n \alpha \sigma}{N-2 \alpha}} v(t) \boldsymbol{D}\right\|_{L^{2}} .
\end{aligned}
$$

Since $\left\|\langle\cdot\rangle^{n} v(t)\right\|_{L^{\infty}} \leq K_{1}$ by (3.20) and (3.12), we deduce from (3.59) and (3.3) that

$$
\begin{aligned}
\left\|\langle\cdot\rangle^{M(|\beta|)-\frac{2 n \alpha \sigma}{N-2 \alpha}} v(t) \boldsymbol{D}^{\mathrm{low}}\right\|_{L^{2}} & \leq C_{0}\left\|\langle\cdot\rangle^{\boldsymbol{M}(|\beta|)-\frac{2 n \alpha \sigma}{N-2 \alpha}-n} \boldsymbol{D}^{\mathrm{low}}\right\|_{L^{2}} \\
& \leq C_{0}\left\|\langle\cdot\rangle^{-\frac{2 n \alpha \sigma}{N-2 \alpha}} \boldsymbol{D}^{\mathrm{low}}\right\|_{L^{2}} \leq C_{0}\left\|\boldsymbol{D}^{\mathrm{low}}\right\|_{L^{\infty}} \\
& \leq \frac{C_{0}}{b}(1-b t)^{-\left(\left|\gamma_{1}\right|-1\right) \sigma} \\
& \leq C_{0}(1-b t)^{-\left(\left|\gamma_{1}\right|-1\right) \sigma} .
\end{aligned}
$$

Estimates (3.65) and (3.66) show that $\boldsymbol{F}$ satisfies (3.26) in the case $\boldsymbol{D}=\boldsymbol{D}^{\text {low }}$. Next, we use (3.3) and (3.61) to obtain

$$
\begin{aligned}
\|\langle\cdot\rangle^{M(|\beta|)-\frac{2 n \alpha \sigma}{N-2 \alpha}} & v(t) \boldsymbol{D}_{2}^{\text {high }}\left\|_{L^{2}} \leq\right\|\langle\cdot\rangle^{M(|\beta|)-2} v(t) \boldsymbol{D}_{2}^{\text {high }} \|_{L^{2}} \\
& \leq \frac{C_{0}}{b}(1-b t)^{-\left(\left|\gamma_{j}\right|-1\right) \sigma} \leq C_{0}(1-b t)^{-\left(\left|\gamma_{j}\right|-1\right) \sigma} .
\end{aligned}
$$

Estimates (3.65) and (3.67) show that $\boldsymbol{F}$ satisfies (3.26) in the case $\boldsymbol{D}=\boldsymbol{D}_{2}^{\text {high }}$. Finally, for $\boldsymbol{D}=\boldsymbol{D}_{1}^{\text {high }}$, we use $\frac{1}{H} \leq 2$ and we obtain

$$
|\boldsymbol{F}| \leq 2\left|D^{\rho}\left(\left|v_{0}(x)\right|^{\alpha}\right)\right|\left|\boldsymbol{D}_{1}^{\mathrm{high}}\right|\left|\prod_{j=2}^{p} \frac{D^{\gamma_{j}} H(t, x)}{H(t, x)}\right| .
$$

We use (2.17) for the first term and (3.62) for the product, and we deduce that

$$
\left\|\langle\cdot\rangle^{M(|\beta|)} v(t) \boldsymbol{F}\right\|_{L^{2}} \leq C_{0}(1-b t)^{-\left(|\beta|-\left|\gamma_{1}\right|-1\right) \sigma}\left\|\langle\cdot\rangle^{-n \alpha+M(|\beta|)} v(t) \boldsymbol{D}_{1}^{\mathrm{high}}\right\|_{L^{2}}
$$

Applying (3.60), we conclude that $\boldsymbol{F}$ also satisfies (3.26) in the case $\boldsymbol{D}=\boldsymbol{D}_{1}^{\text {high }}$. This completes the proof. 


\section{GLOBAL EXISTENCE FOR $\left(\mathrm{NLS}_{b}\right)$}

In this section, we apply Proposition 3.2 and Corollary 3.3 of Section 3 to prove the global existence of solutions to $\left(\mathrm{NLS}_{b}\right)$ for sufficiently large $b$.

The main result of this section is the following.

Proposition 4.1. Let $\lambda \in \mathbb{C}$ satisfy $\Im \lambda<0$. Assume (1.4), (1.5)-(1.7), (3.2) and let $\mathcal{X}$ be defined by (1.10). Let $b>0, K>1$, let $v_{0} \in \mathcal{X}$ satisfy (3.18), let $v \in C\left(\left[0, T_{\max }\right), \mathcal{X}\right)$ be the solution of $\left(\mathrm{NLS}_{b}\right)$ given by Proposition 2.2 , and let $\Psi$ be defined by (3.9)-(3.17). Set

$$
\widetilde{K}=K+\Lambda K^{2}
$$

where $\Lambda$ is the constant in (3.19). It follows that there exists $b_{1} \geq b_{0}>1$, where $b_{0}$ is given by Proposition 3.2, such that if $b \geq b_{1}$, then $T_{\max }=\frac{1}{b}$,

$$
\sup _{0 \leq T<\frac{1}{5}} \Psi_{T} \leq 5 \widetilde{K}
$$

where $\Psi_{T}$ is defined by (3.17), and

$$
\sup _{0 \leq t<T_{\max }} \sup _{x \in \mathbb{R}^{N}}|f(t, x)| \leq \frac{1}{4}
$$

where $f(t, x)$ is defined by (3.34).

For the proof of Proposition 4.1, we will use the following lemma.

Lemma 4.2. Given $b \geq 1, \nu>0, M \geq 1$ and $0<T^{*} \leq \frac{1}{b}$, let

$$
I_{1}(t)=\int_{0}^{t} \min \left\{1,(b G(s))^{1-\frac{2 \sigma}{2-N \alpha}}\right\}(1-b s)^{-\frac{4-N \alpha}{2}-\nu+\sigma} d s,
$$

and

$$
I_{2}(t)=\int_{0}^{t} \min \{M, b G(s)\}(1-b s)^{-\frac{4-N \alpha}{2}-\nu} d s,
$$

$0 \leq t<T^{*}$, where $G$ is defined by (3.23). It follows that there exists a constant $C=C(N, \alpha, \sigma)$ such that

$$
I_{1}(t) \leq \frac{C}{b^{\frac{2 \sigma}{2-N \alpha}}}(1-b t)^{-\nu}
$$

and that

$$
I_{2}(t) \leq\left(\frac{C M}{b^{\frac{2 \sigma}{2-N \alpha}}}+\frac{2}{\nu}\right)(1-b t)^{-\nu}
$$

for all $b \geq 1, M \geq 1$ and $0 \leq t<T^{*}$.

Proof. We first prove (4.6). For a given $b \geq 1$, we consider $T_{b} \in\left(0, \frac{1}{b}\right)$ defined by

$$
b G\left(T_{b}\right)=1 \text {, i.e. }\left(1-b T_{b}\right)^{-\frac{2-N \alpha}{2}}=b+1,
$$

and study two cases separately: the case $0 \leq t \leq T_{b}, t<T^{*}$; and, if $T_{b}<T^{*}$, the case $T_{b} \leq t<T^{*}$.

Suppose first that $0 \leq t \leq T_{b}, t<T^{*}$. Since $\min \left\{1,(b G(s))^{1-\frac{2 \sigma}{2-N \alpha}}\right\}=1$ for $0 \leq s \leq t$, we obtain

$$
I_{1}(t) \leq \int_{0}^{t}(1-b s)^{-\frac{4-N \alpha}{2}-\nu+\sigma} \leq(1-b t)^{-\nu} \int_{0}^{t}(1-b s)^{-\frac{4-N \alpha}{2}+\sigma} .
$$

Note that $-\frac{2-N \alpha}{2}+\sigma<0$ by the third inequality in (3.2), so that using (4.8)

$$
\begin{aligned}
b\left(\frac{2-N \alpha}{2}-\sigma\right) \int_{0}^{t}(1-b s)^{-\frac{4-N \alpha}{2}+\sigma} & =(1-b t)^{-\frac{2-N \alpha}{2}+\sigma}-1 \leq(1-b t)^{-\frac{2-N \alpha}{2}+\sigma} \\
& \leq\left(1-b T_{b}\right)^{-\frac{2-N \alpha}{2}+\sigma}=(b+1)^{1-\frac{2 \sigma}{2-N \alpha}}
\end{aligned}
$$


Thus we see that (4.6) holds for $0 \leq t \leq T_{b}, t<T^{*}$.

Suppose now that $T_{b}<T^{*}$ and $T_{b} \leq t<T^{*}$. Given $s \in\left(t, T^{*}\right)$, we have

$$
\min \left\{1,(b G(s))^{1-\frac{2 \sigma}{2-N \alpha}}\right\}=(b G(s))^{1-\frac{2 \sigma}{2-N \alpha}}=b^{1-\frac{2 \sigma}{2-N \alpha}} G(s)^{1-\frac{2 \sigma}{2-N \alpha}} .
$$

Moreover, by (3.23) and (4.8),

$$
G(s) \leq \frac{(1-b s)^{\frac{2-N \alpha}{2}}}{1-\left(1-b T_{b}\right)^{\frac{2-N \alpha}{2}}}=\frac{(1-b s)^{\frac{2-N \alpha}{2}}}{1-\frac{1}{b+1}} \leq 2(1-b s)^{\frac{2-N \alpha}{2}}
$$

and so

$$
\min \left\{1,(b G(s))^{1-\frac{2 \sigma}{2-N \alpha}}\right\} \leq 2 b^{1-\frac{2 \sigma}{2-N \alpha}}(1-b s)^{\frac{2-N \alpha}{2}-\sigma} .
$$

Applying (4.6) with $T=T_{b}$, we deduce that

$$
\begin{aligned}
I_{1}(t) & \leq \frac{C}{b^{\frac{2 \sigma}{2-N \alpha}}}\left(1-b T_{b}\right)^{-\nu}+2 b^{1-\frac{2 \sigma}{2-N \alpha}} \int_{T_{b}}^{t}(1-b s)^{-1-\nu} \\
& \leq \frac{C}{b^{\frac{2 \sigma}{2-N \alpha}}}(1-b t)^{-\nu}+2 b^{1-\frac{2 \sigma}{2-N \alpha}} \int_{0}^{t}(1-b s)^{-1-\nu} \\
& \leq \frac{C}{b^{\frac{2 \sigma}{2-N \alpha}}}(1-b t)^{-\nu} .
\end{aligned}
$$

Thus (4.6) is satisfied for all $0 \leq t<T^{*}$.

We now prove (4.8). For a given $b \geq 1$, we consider $\widetilde{T}_{b} \in\left(0, \frac{1}{b}\right)$ defined by

$$
\left(1-b \widetilde{T}_{b}\right)^{-\frac{2-N \alpha}{2}}=1+b^{1-\frac{2 \sigma}{2-N \alpha}},
$$

and study two cases separately: the case $0 \leq t \leq \widetilde{T}_{b}, t<T^{*}$; and, if $\widetilde{T}_{b}<T^{*}$ the case $\widetilde{T}_{b} \leq t<T^{*}$

Suppose first that $0 \leq t \leq \widetilde{T}_{b}, t<T^{*}$. Since $\min \left\{M,(b G(s))^{1-\frac{2 \sigma}{2-N \alpha}}\right\} \leq M$, we obtain

$$
\begin{aligned}
I_{2}(t) & \leq M \int_{0}^{t}(1-b s)^{-\frac{4-N \alpha}{2}-\nu} \leq M(1-b t)^{-\nu} \int_{0}^{t}(1-b s)^{-\frac{4-N \alpha}{2}} \\
& \leq M(1-b t)^{-\nu} \frac{2}{(2-N \alpha) b}\left[(1-b t)^{-\frac{2-N \alpha}{2}}-1\right],
\end{aligned}
$$

so that

$$
I_{2}(t) \leq \frac{2 M}{(2-N \alpha) b^{\frac{2 \sigma}{2-N \alpha}}}(1-b t)^{-\nu} .
$$

Suppose now that $\widetilde{T}_{b}<T^{*}$ and $\widetilde{T}_{b} \leq t<T^{*}$. Given $s \in\left(t, T^{*}\right)$, we have by $(3.23)$ and (4.10),

$$
G(s) \leq \frac{(1-b s)^{\frac{2-N \alpha}{2}}}{1-\left(1-b \widetilde{T}_{b}\right)^{\frac{2-N \alpha}{2}}}=\frac{(1-b s)^{\frac{2-N \alpha}{2}}}{1-\frac{1}{1+b^{1-\frac{2 \sigma}{2-N \alpha}}}} \leq 2(1-b s)^{\frac{2-N \alpha}{2}} .
$$

It follows that

$$
\int_{\widetilde{T}_{b}}^{t} b G(s)(1-b s)^{-\frac{4-N \alpha}{2}-\nu} \leq 2 b \int_{\widetilde{T}_{b}}^{t}(1-b s)^{-1-\nu} \leq \frac{2}{\nu}(1-b t)^{-\nu} .
$$

Applying (4.11) with $t=\widetilde{T}_{b}$, we conclude that

$$
I_{2}(t) \leq\left(\frac{2 M}{(2-N \alpha) b^{\frac{2 \sigma}{2-N \alpha}}}+\frac{2}{\nu}\right)(1-b t)^{-\nu} .
$$

This, together with (4.11), proves (4.8). 
Proof of Proposition 4.1. It follows from (4.1) and Lemma 3.1 that $\Psi_{0} \leq \widetilde{K}$ and that $\Psi_{t} \leq 2 \widetilde{K}$ for $t>0$ and small. We set

$$
T^{*}=\sup \left\{0 \leq T<T_{\max } ; \Psi_{T} \leq 5 \widetilde{K}\right\}
$$

so that

$$
0<T^{*} \leq T_{\max }
$$

We claim that there exists $b_{1} \geq b_{0}$, such that if $b \geq b_{1}$ then

$$
T^{*}=T_{\max } \text {. }
$$

Assuming the claim (4.13), we complete the proof. First, if $T_{\max }<\frac{1}{b}$, then by (4.12), (4.13) and Lemma 3.1, we see that $\Psi_{T_{\max }} \leq 5 \widetilde{K}$. It follows that for all $0 \leq t<T_{\max }$

$$
\|v(t)\|_{\mathcal{X}}+\left(\inf _{x \in \mathbb{R}^{N}}\langle x\rangle^{n}|v(t, x)|\right)^{-1} \leq 5 \widetilde{K}(1-b t)^{-\sigma_{J}} \leq 5 \widetilde{K}\left(1-b T_{\max }\right)^{-\sigma_{J}}<\infty
$$

which contradicts the blowup alternative (2.11). Thus $T^{*}=T_{\max }=\frac{1}{b}$, and then estimate (4.2) follows from (4.12). Moreover, applying (3.39) with $K_{1}=5 \widetilde{K}$, we deduce that (4.3) holds, by choosing $b_{1}$ possibly larger.

We prove the claim (4.13) by contradiction, so we assume that

$$
T^{*}<T_{\max }
$$

By the definition of $T^{*}$ and continuity of $\Psi_{T}$ in $T$, we see that

$$
\Psi_{T^{*}}=5 \widetilde{K}
$$

In the rest of the proof, we apply Proposition 3.2 and Corollary 3.3 with $T=T^{*}$ and $K_{1}=5 \widetilde{K}$, and in particular we assume $b \geq b_{0}$. For further reference, we note that by (3.22),

$$
\alpha\|v(t)\|_{L^{\infty}}^{\alpha} \leq\left(\frac{1+\alpha|\Im \lambda|}{|\Im \lambda|}\right) \min \left\{2 K^{\alpha}, b G(t)\right\}
$$

for all $0 \leq t \leq T^{*}$. Moreover, we denote by $C_{1}, C_{2}, C_{3}, C_{4}, C_{5}>0$ various constants depending possibly on $\beta, \alpha, N, K, K_{1}, \lambda$, etc, but not on $b, v, T^{*}$, whose exact values are irrelevant and can change from line to line.

We proceed in several steps.

SteP 1. Control of $\Phi_{1, T^{*}}$. It follows from (3.21) that

$$
\left\|\langle\cdot\rangle^{n} v(t)\right\|_{L^{\infty}} \leq 2\left\|\langle\cdot\rangle^{n} v_{0}\right\|_{L^{\infty}} \leq 2 \widetilde{K}
$$

Next, given $1 \leq|\beta| \leq 2 m$, we apply (2.3) or (2.4) with $f(s)=\lambda(1-b s)^{-\frac{4-N \alpha}{2}}|v|^{\alpha} v$ to obtain

$$
\left|\langle x\rangle^{n} D^{\beta} v\right| \leq \widetilde{K}+I+\int_{0}^{t}(1-b s)^{-\frac{4-N \alpha}{2}} \Im\left(\lambda \frac{\langle x\rangle^{2 n} D^{\beta}\left(|v|^{\alpha} v\right) D^{\beta} \bar{v}}{\langle x\rangle^{n}\left|D^{\beta} v\right|}\right) d s,
$$

where

$$
I= \begin{cases}\int_{0}^{t} \sup _{|\gamma| \leq|\beta|+2}\left\|\langle\cdot\rangle^{n} D^{\gamma} v(s)\right\|_{L^{\infty}} d s, & |\beta| \leq 2 m-2, \\ A \int_{0}^{t} \sup _{|\beta|+2 \leq|\gamma| \leq|\beta|+k+2}\left\|\langle\cdot\rangle^{n} D^{\gamma} v(s)\right\|_{L^{2}} d s, & 2 m-1 \leq|\beta| \leq 2 m .\end{cases}
$$

It follows from (4.14), (3.12), (3.13) and (3.7) that

$$
I \leq 5 \widetilde{K}(1+A) \int_{0}^{t}(1-b s)^{-\sigma(|\beta|+k+4)} .
$$


Since $\sigma(k+4)<1$ by the fourth inequality in (3.2) and $\sigma|\beta|=\sigma_{|\beta|}$, we obtain

$$
I \leq 5 \widetilde{K}(1+A)(1-b t)^{-\sigma_{|\beta|}} \int_{0}^{\frac{1}{b}}(1-b s)^{-\sigma(k+4)} \leq \frac{C_{1}}{b}(1-b t)^{-\sigma_{|\beta|}} .
$$

To estimate the last term in (4.17), we write

$$
D^{\beta}\left(|v|^{\alpha} v\right)=|v|^{\alpha} D^{\beta} v+\sum_{\substack{\gamma_{1}+\gamma_{2}=\beta \\\left|\gamma_{1}\right| \geq 1}} c_{\gamma_{1}, \gamma_{2}} D^{\gamma_{1}}\left(|v|^{\alpha}\right) D^{\gamma_{2}} v
$$

where the coefficients $c_{\gamma_{1}, \gamma_{2}}$ are given by Leibniz's rule. Since

$$
\Im\left(\lambda|v|^{\alpha} D^{\beta} v D^{\beta} \bar{v}\right)=(\Im \lambda)|v|^{\alpha}\left|D^{\beta} v\right|^{2} \leq 0
$$

we see that

Moreover,

$$
\Im\left(\lambda \frac{\langle x\rangle^{2 n}|v|^{\alpha} D^{\beta} v D^{\beta} \bar{v}}{\langle x\rangle^{n}\left|D^{\beta} v\right|}\right) \leq 0
$$

$$
\left|\lambda \frac{\langle x\rangle^{2 n} D^{\gamma_{1}}\left(|v|^{\alpha}\right) D^{\gamma_{2}} v D^{\beta} \bar{v}}{\langle x\rangle^{n}\left|D^{\beta} v\right|}\right| \leq|\lambda|\langle x\rangle^{n}\left|D^{\gamma_{1}}\left(|v|^{\alpha}\right)\right|\left|D^{\gamma_{2}} v\right|
$$

so that

$$
\Im\left(\lambda \frac{\langle x\rangle^{2 n} D^{\beta}\left(|v|^{\alpha} v\right) D^{\beta} \bar{v}}{\langle x\rangle^{n}\left|D^{\beta} v\right|}\right) \leq C_{1} \sum_{\substack{\gamma_{1}+\gamma_{2}=\beta \\\left|\gamma_{1}\right| \geq 1}}\left\|\langle\cdot\rangle^{n} D^{\gamma_{1}}\left(|v|^{\alpha}\right) D^{\gamma_{2}} v\right\|_{L^{\infty}} .
$$

Applying (4.14), (3.25) (with $\beta$ replaced by $\gamma_{1}$, recall that $\left|\gamma_{1}\right| \geq 1$ ), (3.12) and (3.7), we obtain

$$
\left\|\langle\cdot\rangle^{n} D^{\gamma_{1}}\left(|v|^{\alpha}\right) D^{\gamma_{2}} v\right\|_{L^{\infty}} \leq C_{1} \min \left\{1,(b G(s))^{1-\frac{2 \sigma}{2-N \alpha}}\right\}(1-b s)^{-(|\beta|-1) \sigma} .
$$

Applying (4.6) of Lemma 4.2 with $\nu=|\beta| \sigma$, we deduce that

$$
\int_{0}^{t}(1-b s)^{-\frac{4-N \alpha}{2}}\left\|\langle\cdot\rangle^{n} D^{\gamma_{1}}\left(|v|^{\alpha}\right) D^{\gamma_{2}} v\right\|_{L^{\infty}} \leq \frac{C_{1}}{b^{\frac{2 \sigma}{2-N \alpha}}}(1-b t)^{-|\beta| \sigma} .
$$

Estimates (4.21) and (4.22) prove that

$$
\int_{0}^{t}(1-b s)^{-\frac{4-N \alpha}{2}} \Im\left(\lambda \frac{\langle x\rangle^{2 n} D^{\beta}\left(|v|^{\alpha} v\right) D^{\beta} \bar{v}}{\langle x\rangle^{n}\left|D^{\beta} v\right|}\right) d s \leq \frac{C_{1}}{b^{\frac{2 \sigma}{2-N \alpha}}}(1-b t)^{-|\beta| \sigma},
$$

for all $0 \leq t \leq T^{*}$. Since $|\beta| \sigma=\sigma_{|\beta|}$, it now follows from (4.16), (4.17), (4.18), and (4.23) that if $b_{1} \geq b_{0}$ is sufficiently large so that

$$
C_{1}\left(\frac{1}{b}_{1}+\frac{1}{b_{1}^{\frac{2 \sigma}{2-N \alpha}}}\right)<\widetilde{K}
$$

then

$$
\Phi_{1, T^{*}} \leq 2 \widetilde{K}
$$

provided $b \geq b_{1}$.

STEP 2. Control of $(1-b t)^{\sigma|\beta|}\left\|\langle x\rangle^{M(|\beta|)} D^{\beta} v\right\|_{L^{2}}$ for $2 m+1 \leq|\beta| \leq J-2$. Applying (2.5) with $\mu=n-\boldsymbol{M}(|\beta|) \in[0, n]$ (which corresponds to $\nu=|\beta|-2 m-1 \leq$ $k+1$ if $\boldsymbol{M}(|\beta|)=n$ and $\nu=k+1$ if $\boldsymbol{M}(|\beta|)<n)$ and $f(s)=\lambda(1-b s)^{-\frac{4-N \alpha}{2}}|v|^{\alpha} v$ we obtain

$$
\left\|\langle\cdot\rangle^{M(|\beta|)} D^{\beta} v\right\|_{L^{2}} \leq \widetilde{K}+C_{2} \int_{0}^{t}\left\|\langle\cdot\rangle^{M(|\beta|)-1} \nabla D^{\beta} v\right\|_{L^{2}} d s+\widetilde{I},
$$

where

$$
\widetilde{I}=\int_{0}^{t}(1-b s)^{-\frac{4-N \alpha}{2}} \frac{\Im \int_{\mathbb{R}^{N}} \lambda\langle x\rangle^{2 M(|\beta|)} D^{\beta}\left(|v|^{\alpha} v\right) D^{\beta} \bar{v} d x}{\left\|\langle\cdot\rangle^{M(|\beta|)} D^{\beta} v\right\|_{L^{2}}} d s
$$


Note that $\boldsymbol{M}(|\beta|)-1 \leq \boldsymbol{M}(|\beta|+1)$. Therefore, it follows from (4.14), (3.13) and (3.7) that

$$
\int_{0}^{t}\left\|\langle\cdot\rangle^{M(|\beta|)-1} \nabla D^{\beta} v\right\|_{L^{2}} d s \leq 5 \widetilde{K} \int_{0}^{t}(1-b s)^{-(|\beta|+3) \sigma} d s .
$$

Since $2 \sigma<1$ by the fourth inequality in (3.2), we deduce that

$$
\begin{aligned}
\int_{0}^{t}(1-b s)^{-(|\beta|+3) \sigma} d s & \leq(1-b t)^{-(|\beta|+1) \sigma} \int_{0}^{t}(1-b s)^{-2 \sigma} d s \\
& \leq \frac{C_{2}}{b}(1-b t)^{-(|\beta|+1) \sigma} \leq \frac{C_{2}}{b}(1-b t)^{-\sigma_{|\beta|}} .
\end{aligned}
$$

Thus we conclude that

$$
C_{2} \int_{0}^{t}\left\|\langle\cdot\rangle^{M(|\beta|)-1} \nabla D^{\beta} v\right\|_{L^{2}} d s \leq \frac{C_{2}}{b}(1-b t)^{-\sigma_{|\beta|}} .
$$

To estimate $\widetilde{I}$ we use, as in Step 1, (4.19) and (4.20) and we deduce that

$$
\begin{aligned}
\Im \int_{\mathbb{R}^{N}} \lambda\langle x\rangle^{2 M(|\beta|)} D^{\beta}\left(|v|^{\alpha} v\right) D^{\beta} \bar{v} d x \\
\leq C_{2} \sum_{\substack{\gamma_{1}+\gamma_{2}=\beta \\
\left|\gamma_{1}\right| \geq 1}}\left\|\langle\cdot\rangle^{2 M(|\beta|)} D^{\gamma_{1}}\left(|v|^{\alpha}\right) D^{\gamma_{2}} v D^{\beta} \bar{v}\right\|_{L^{1}} \\
\leq C_{2}\left\|\langle\cdot\rangle^{M(|\beta|)} D^{\beta} v\right\|_{L^{2}} \sum_{\substack{\gamma_{1}+\gamma_{2}=\beta \\
\left|\gamma_{1}\right| \geq 1}}\left\|\langle\cdot\rangle^{M(|\beta|)} D^{\gamma_{1}}\left(|v|^{\alpha}\right) D^{\gamma_{2}} v\right\|_{L^{2}} ;
\end{aligned}
$$

and so,

$$
\begin{aligned}
& \frac{\Im \int_{\mathbb{R}^{N}} \lambda\langle x\rangle^{2 M(|\beta|)} D^{\beta}\left(|v|^{\alpha} v\right) D^{\beta} \bar{v} d x}{\left\|\langle\cdot\rangle^{M(|\beta|)} D^{\beta} v\right\|_{L^{2}}} \\
& \quad \leq C_{2} \sum_{\substack{\gamma_{1}+\gamma_{2}=\beta \\
\left|\gamma_{1}\right| \geq 1}}\left\|\langle\cdot\rangle^{M(|\beta|)} D^{\gamma_{1}}\left(|v|^{\alpha}\right) D^{\gamma_{2}} v\right\|_{L^{2}} .
\end{aligned}
$$

To estimate the right-hand side of (4.29), we distinguish three cases: $\left|\gamma_{2}\right| \geq 2 m+1$; $\left|\gamma_{2}\right| \leq 2 m$ and $\left|\gamma_{1}\right| \geq 2 m+1 ;\left|\gamma_{2}\right| \leq 2 m$ and $\left|\gamma_{1}\right| \leq 2 m$. If $\left|\gamma_{2}\right| \geq 2 m+1$, then $\left|\gamma_{1}\right| \leq 2 m$. (Indeed, $\left|\gamma_{1}\right|+\left|\gamma_{2}\right|=|\beta| \leq J \leq 4 m+1$, by definition of $J$ and the first inequality in (1.7).) Since $\boldsymbol{M}(|\beta|) \leq \boldsymbol{M}\left(\left|\gamma_{2}\right|\right)$, we have

$$
\left\|\langle\cdot\rangle^{M(|\beta|)} D^{\gamma_{1}}\left(|v|^{\alpha}\right) D^{\gamma_{2}} v\right\|_{L^{2}} \leq\left\|D^{\gamma_{1}}\left(|v|^{\alpha}\right)\right\|_{L^{\infty}}\left\|\langle\cdot\rangle^{M\left(\left|\gamma_{2}\right|\right)} D^{\gamma_{2}} v\right\|_{L^{2}} .
$$

It follows from (3.25) (with $\beta$ replaced by $\gamma_{1}$, recall that $\left|\gamma_{1}\right| \geq 1$ ), and from (4.14), (3.13), (3.7), that

$$
\left\|\langle\cdot\rangle^{M(|\beta|)} D^{\gamma_{1}}\left(|v|^{\alpha}\right) D^{\gamma_{2}} v\right\|_{L^{2}} \leq C_{2} \min \left\{1,(b G(s))^{1-\frac{2 \sigma}{2-N \alpha}}\right\}(1-b s)^{-(|\beta|+1) \sigma} .
$$

(We use the fact that since $\left|\gamma_{2}\right| \leq J-2, \sigma_{\left|\gamma_{2}\right|} \leq\left(\left|\gamma_{2}\right|+2\right) \sigma$.) If $\left|\gamma_{2}\right| \leq 2 m$ and $\left|\gamma_{1}\right| \geq 2 m+1$, we deduce from (3.27) (with $\beta$ replaced by $\gamma_{1}$ and $\gamma$ by $\gamma_{2}$ ) and the property $\boldsymbol{M}(|\beta|) \leq \boldsymbol{M}\left(\left|\gamma_{1}\right|\right)$ that estimate (4.30) also holds. If $\left|\gamma_{1}\right|,\left|\gamma_{2}\right| \leq 2 m$, we use the fact that $\langle x\rangle^{-\frac{2 n \alpha \sigma}{2-N \alpha}} \in L^{2}\left(\mathbb{R}^{N}\right)$ by (3.3), and we estimate

$$
\begin{aligned}
\left\|\langle\cdot\rangle^{M(|\beta|)} D^{\gamma_{1}}\left(|v|^{\alpha}\right) D^{\gamma_{2}} v\right\|_{L^{2}} & \leq\left\|D^{\gamma_{1}}\left(|v|^{\alpha}\right)\right\|_{L^{2}}\left\|\langle\cdot\rangle^{n} D^{\gamma_{2}} v\right\|_{L^{\infty}} \\
& \leq C_{2}\left\|\langle\cdot\rangle^{\frac{2 n \alpha \sigma}{2-N \alpha}} D^{\gamma_{1}}\left(|v|^{\alpha}\right)\right\|_{L^{\infty}}\left\|\langle\cdot\rangle^{n} D^{\gamma_{2}} v\right\|_{L^{\infty}} .
\end{aligned}
$$

Using (3.25), and (4.14), (3.12) and (3.7) we see that in this case also estimate (4.30) holds. Applying (4.30) and (4.6) of Lemma 4.2 with $\nu=(|\beta|+2) \sigma$, we deduce that

$$
\widetilde{I} \leq \frac{C_{2}}{b^{\frac{2 \sigma}{2-N \alpha}}}(1-b t)^{-(|\beta|+2) \sigma} .
$$


Since $(|\beta|+2) \sigma \geq \sigma_{|\beta|}$, it now follows from (4.26), (4.28) and (4.31) that $b_{1} \geq b_{0}$ is sufficiently large so that

$$
C_{2}\left(\frac{1}{b}_{1}+\frac{1}{b_{1}^{\frac{2 \sigma}{2-N \alpha}}}\right)<\widetilde{K}
$$

then

$$
\sup _{2 m+1 \leq|\beta| \leq J-2} \sup _{0 \leq t \leq T^{*}}(1-b t)^{\sigma|\beta|}\left\|\langle\cdot\rangle^{M(|\beta|)} D^{\beta} v\right\|_{L^{2}} \leq 2 \widetilde{K}
$$

provided $b \geq b_{1}$.

STEP 3. Control of $(1-b t)^{\sigma_{|\beta|}}\left\|\langle x\rangle^{M(|\beta|)} D^{\beta} v\right\|_{L^{2}}$ for $J-1 \leq|\beta| \leq J$. Applying (2.5) with $\mu=n-\boldsymbol{M}(|\beta|)=n-(J-|\beta|) \in\{n-1, n\}, \nu=k+1$, and $f(s)=\lambda(1-b s)^{-\frac{4-N \alpha}{2}}|v|^{\alpha} v$ we obtain

$$
\left\|\langle\cdot\rangle^{M(|\beta|)} D^{\beta} v\right\|_{L^{2}} \leq \widetilde{K}+C_{2}(J-|\beta|) \int_{0}^{t}\left\|\langle\cdot\rangle^{J-1-|\beta|} \nabla D^{\beta} v\right\|_{L^{2}} d s+\widetilde{I},
$$

where

$$
\widetilde{I}=\int_{0}^{t}(1-b s)^{-\frac{4-N \alpha}{2}} \frac{\Im \int_{\mathbb{R}^{N}} \lambda\langle x\rangle^{2 \boldsymbol{M}(|\beta|)} D^{\beta}\left(|v|^{\alpha} v\right) D^{\beta} \bar{v} d x}{\left\|\langle\cdot\rangle^{\boldsymbol{M}(|\beta|)} D^{\beta} v\right\|_{L^{2}}} d s
$$

The second term in the right-hand side of (4.34) vanishes if $|\beta|=J$, so we need only estimate it if $|\beta|=J-1$. In this case, it follows from (4.14), (3.13) and (3.7)

$$
\begin{aligned}
\int_{0}^{t}\left\|\langle\cdot\rangle^{J-1-|\beta|} \nabla D^{\beta} v\right\|_{L^{2}} d s & =\int_{0}^{t}\left\|\nabla D^{\beta} v\right\|_{L^{2}} \\
& \leq 5 \widetilde{K} \int_{0}^{t}(1-b s)^{-(|\beta|+5) \sigma} d s .
\end{aligned}
$$

Since $2 \sigma<1$ by the fourth inequality in (3.2), we see that (compare (4.27))

$$
\int_{0}^{t}(1-b s)^{-(|\beta|+5) \sigma} d s \leq \frac{C_{3}}{b}(1-b t)^{-(|\beta|+3) \sigma}=\frac{C_{3}}{b}(1-b t)^{-\sigma_{|\beta|}} .
$$

Thus we obtain

$$
C_{2}(J-|\beta|) \int_{0}^{t}\left\|\langle\cdot\rangle^{J-1-|\beta|} \nabla D^{\beta} v\right\|_{L^{2}} d s \leq \frac{C_{3}}{b}(1-b t)^{-\sigma_{|\beta|}},
$$

for both $|\beta|=J-1$ and $|\beta|=J$.

To estimate $\widetilde{I}$ we use a more precise version of (4.19) where we isolate the term corresponding to $\gamma_{1}=\beta$, i.e.

$$
D^{\beta}\left(|v|^{\alpha} v\right)=|v|^{\alpha} D^{\beta} v+D^{\beta}\left(|v|^{\alpha}\right) v+c_{\gamma_{1}, \gamma_{2}} \sum_{\substack{\gamma_{1}+\gamma_{2}=\beta \\\left|\gamma_{1}\right|,\left|\gamma_{2}\right| \geq 1}} D^{\gamma_{1}}\left(|v|^{\alpha}\right) D^{\gamma_{2}} v .
$$

Using (4.20), we obtain (compare (4.29))

$$
\begin{gathered}
\frac{\Im \int_{\mathbb{R}^{N}} \lambda\langle x\rangle^{2 M(|\beta|)} D^{\beta}\left(|v|^{\alpha} v\right) D^{\beta} \bar{v} d x}{\left\|\langle\cdot\rangle^{M(|\beta|)} D^{\beta} v\right\|_{L^{2}}} \leq|\lambda|\left\|\langle\cdot\rangle^{M(|\beta|)} D^{\beta}\left(|v|^{\alpha}\right) v\right\|_{L^{2}} \\
+C_{3} \sum_{\substack{\gamma_{1}+\gamma_{2}=\beta \\
\left|\gamma_{1}\right|,\left|\gamma_{2}\right| \geq 1}}\left\|\langle\cdot\rangle^{M(|\beta|)} D^{\gamma_{1}}\left(|v|^{\alpha}\right) D^{\gamma_{2}} v\right\|_{L^{2}} .
\end{gathered}
$$

For the first term on the right-hand side of (4.37), we use formula (2.15) with $\rho$ replaced by $\alpha$, and we obtain

$$
\begin{aligned}
|\lambda|\left\|\langle\cdot\rangle^{M(|\beta|)} D^{\beta}\left(|v|^{\alpha}\right) v\right\|_{L^{2}} \leq & \lambda\left|\alpha\left\|\langle\cdot\rangle^{M(|\beta|)}|v|^{\alpha} D^{\beta} v\right\|_{L^{2}}\right. \\
& +C_{3} \sup _{D}\left\|\langle\cdot\rangle^{M(|\beta|)}|v|^{\alpha+1} \prod_{\ell=1}^{|\beta|} \frac{\left|D^{\beta_{\ell}} v\right|}{|v|}\right\|_{L^{2}},
\end{aligned}
$$


where $D$ is the set of $\left(\beta_{\ell}\right)_{1 \leq \ell \leq|\beta|}$ with $0 \leq\left|\beta_{\ell}\right| \leq|\beta|-1$ and $\sum_{\ell=1}^{|\beta|} \beta_{\ell}=\beta$. It follows from (4.15) and (4.14) that

$$
\begin{aligned}
|\lambda| \alpha\left\|\langle\cdot\rangle^{M(|\beta|)}|v|^{\alpha} D^{\beta} v\right\|_{L^{2}} & \leq|\lambda| \alpha\|v\|_{L^{\infty}}^{\alpha}\left\|\langle\cdot\rangle^{M(|\beta|)} D^{\beta} v\right\|_{L^{2}} \\
& \leq 5|\lambda| \widetilde{K}\left(\frac{1+\alpha|\Im \lambda|}{|\Im \lambda|}\right) \min \left\{2 K^{\alpha}, b G(t)\right\}(1-b s)^{-\sigma_{|\beta|}} .
\end{aligned}
$$

Applying (4.7) of Lemma 4.2 with $\nu=\sigma_{|\beta|}$ and $M=2 K^{\alpha}$, and then (3.8), we deduce that

$$
\begin{aligned}
& \int_{0}^{t}(1-b s)^{-\frac{4-N \alpha}{2}}|\lambda| \alpha\left\|\langle\cdot\rangle^{M(|\beta|)}|v|^{\alpha} D^{\beta} v\right\|_{L^{2}} d s \\
& \leq 5|\lambda| \widetilde{K}\left(\frac{1+\alpha|\Im \lambda|}{|\Im \lambda|}\right)\left(\frac{2 C K^{\alpha}}{b^{\frac{2 \sigma}{2-N \alpha}}}+\frac{2}{\sigma_{|\beta|}}\right)(1-b t)^{-\nu} \\
& \leq\left[5|\lambda| \widetilde{K}\left(\frac{1+\alpha|\Im \lambda|}{|\Im \lambda|}\right)\left(\frac{2 C K^{\alpha}}{b^{\frac{2 \sigma}{2-N \alpha}}}\right)+\widetilde{K}\right](1-b t)^{-\nu} \\
& \leq\left(\frac{C_{3}}{b^{\frac{2 \sigma}{2-N \alpha}}}+\widetilde{K}\right)(1-b t)^{-\nu} .
\end{aligned}
$$

We next consider the last terms in the right-hand side of (4.37), and we consider separately the cases $\left|\gamma_{1}\right| \leq J-2$ and $J-1 \leq\left|\gamma_{1}\right| \leq J$. (Indeed, if $\left|\gamma_{1}\right| \geq J-1$, then we may not apply estimate (3.27) with $\beta$ replaced by $\gamma_{1}$ as we do in Step 2.) If $\left|\gamma_{1}\right| \leq J-2$, then we may proceed as in Step 2 and obtain, similarly to (4.30) and (4.31), then using $(|\beta|+2) \sigma \leq \sigma_{|\beta|}-\sigma$

$$
\begin{aligned}
\int_{0}^{t}(1-b s)^{-\frac{4-N \alpha}{2}}\left\|\langle\cdot\rangle^{M(|\beta|)} D^{\gamma_{1}}\left(|v|^{\alpha}\right) D^{\gamma_{2}} v\right\|_{L^{2}} d s & \leq \frac{C_{3}}{b^{\frac{2 \sigma}{2-N \alpha}}}(1-b t)^{-(|\beta|+2) \sigma} \\
& \leq \frac{C_{3}}{b^{\frac{2 \sigma}{2-N \alpha}}}(1-b t)^{-\sigma_{|\beta|}} .
\end{aligned}
$$

We now consider the case $J-1 \leq\left|\gamma_{1}\right| \leq J$. Applying formula (2.15) with $\beta$ replaced by $\gamma_{1}$ and $\rho$ by $\alpha$, we obtain

$$
\frac{\left|D^{\gamma_{1}}\left(|v|^{\alpha}\right)\right|}{|v|^{\alpha}} \leq C_{3} \sup _{\widetilde{D}} \prod_{\ell=1}^{\left|\gamma_{1}\right|} \frac{\left|D^{\beta_{\ell}} v\right|}{|v|}
$$

where $\widetilde{D}$ is the set of $\left(\beta_{\ell}\right)_{1 \leq \ell \leq\left|\gamma_{1}\right|}$ with $\left.0 \leq\left|\beta_{\ell}\right| \leq\left|\gamma_{1}\right|\right)$ and $\sum_{\ell=1}^{\left|\gamma_{1}\right|} \beta_{\ell}=\gamma_{1}$. Thus we see that we have to estimate terms of the form

$$
\boldsymbol{A}=\left\|\langle\cdot\rangle^{\boldsymbol{M}(|\beta|)}|v|^{\alpha}\left|D^{\gamma_{2}} v\right| \prod_{\ell=1}^{\left|\gamma_{1}\right|} \frac{\left|D^{\beta_{\ell}} v\right|}{|v|}\right\|_{L^{2}},
$$

where $\left.\gamma_{1}+\gamma_{2}=\beta, 0 \leq\left|\beta_{\ell}\right| \leq|\beta|-1\right)$ and $\sum_{\ell=1}^{\left|\gamma_{1}\right|} \beta_{\ell}=\gamma_{1}$. If all $\left|\beta_{\ell}\right| \leq 2 m$, then writing

$$
\boldsymbol{A} \leq\left\||v|^{\alpha}\right\|_{L^{2}}\left\|\langle\cdot\rangle^{n} D^{\gamma_{2}} v\right\|_{L^{\infty}} \prod_{\ell=1}^{\left|\gamma_{1}\right|}\left\|\frac{\left|D^{\beta_{\ell}} v\right|}{|v|}\right\|_{L^{\infty}},
$$

we deduce from (3.24), (4.14), (3.12), (3.15) and (3.7) that

$$
\boldsymbol{A} \leq C_{3} \min \left\{1,(b G(s))^{1-\frac{2 \sigma}{2-N \alpha}}\right\}(1-b s)^{-|\beta| \sigma} .
$$

Since $|\beta| \geq J-1$, we have $|\beta| \sigma \leq \sigma_{|\beta|}-\sigma$, so that

$$
\boldsymbol{A} \leq C_{3} \min \left\{1,(b G(s))^{1-\frac{2 \sigma}{2-N \alpha}}\right\}(1-b s)^{-\sigma_{|\beta|}+\sigma} .
$$


If one the $\left|\beta_{\ell}\right| \geq 2 m+1$, say $\left|\beta_{1}\right| \geq 2 m+1$, then $\left|\beta_{\ell}\right| \leq 2 m$ for $\ell \geq 2$. Writing

$$
\boldsymbol{A} \leq\left\||v|^{\alpha}\right\|_{L^{\infty}}\left\|\frac{\left|D^{\gamma_{2}} v\right|}{|v|}\right\|_{L^{\infty}}\left\|\langle\cdot\rangle^{M\left(\left|\beta_{1}\right|\right)} D^{\beta_{1}} v\right\|_{L^{2}} \prod_{\ell=2}^{\left|\gamma_{1}\right|}\left\|\frac{\left|D^{\beta_{\ell}} v\right|}{|v|}\right\|_{L^{\infty}},
$$

(where we use $\boldsymbol{M}(|\beta|) \leq \boldsymbol{M}\left(\left|\beta_{1}\right|\right)$ ), we deduce from (3.22), (4.14), (3.13), (3.15) and (3.7) that

$$
\boldsymbol{A} \leq C_{3} \min \{1,(b G(s))\}(1-b s)^{-\left|\gamma_{2}\right| \sigma-\sigma_{\left|\beta_{1}\right|}-\left(\sum_{\ell=2}^{\left|\gamma_{1}\right|}\left|\beta_{\ell}\right|\right) \sigma} .
$$

Since $\min \{1,(b G(s))\} \leq \min \left\{1,(b G(s))^{1-\frac{2 \sigma}{2-N \alpha}}\right\}$ and $\sum_{\ell=2}^{\left|\gamma_{1}\right|}\left|\beta_{\ell}\right|=\left|\gamma_{1}\right|-\left|\beta_{1}\right|$, we see that

$$
\boldsymbol{A} \leq C_{3} \min \{1,(b G(s))\}(1-b s)^{-|\beta| \sigma-\sigma_{\left|\beta_{1}\right|+\left|\beta_{1}\right| \sigma} .}
$$

If $|\beta|=J$, then $\sigma_{|\beta|}=(|\beta|+4) \sigma$, and $\left|\beta_{1}\right| \leq|\beta|-1$, so that $\sigma_{\left|\beta_{1}\right|}-\left|\beta_{1}\right| \sigma \leq 3 \sigma$; and so $-|\beta| \sigma-\sigma_{\left|\beta_{1}\right|}+\left|\beta_{1}\right| \sigma \geq-\sigma_{|\beta|}+\sigma$. If $|\beta|=J-1$, then $\sigma_{|\beta|}=(|\beta|+3) \sigma$, and $\left|\beta_{1}\right| \leq|\beta|-1$, so that $\sigma_{\left|\beta_{1}\right|}-\left|\beta_{1}\right| \sigma \leq 2 \sigma$; and so $-|\beta| \sigma-\sigma_{\left|\beta_{1}\right|}+\left|\beta_{1}\right| \sigma \geq-\sigma_{|\beta|}+\sigma$. In both cases, we see that $\boldsymbol{A}$ satisfies the estimate (4.41). Applying (4.41) and (4.6) of Lemma 4.2 with $\nu=\sigma_{|\beta|}$, we deduce that

$$
\int_{0}^{t}(1-b s)^{-\frac{4-N \alpha}{2}} \boldsymbol{A} d s \leq \frac{C_{3}}{b^{\frac{2 \sigma}{2-N \alpha}}}(1-b t)^{-\sigma_{|\beta|}} .
$$

Note also that the last terms in (4.38) are of the form $\boldsymbol{A}$ with $\left|\gamma_{2}\right|=0$, so they also satisfy (4.42). Applying (4.37), (4.38), (4.39), (4.40) and (4.42), we deduce that

$$
\widetilde{I} \leq\left(\widetilde{K}+\frac{C_{3}}{b^{\frac{2 \sigma}{2-N \alpha}}}\right)(1-b t)^{-\sigma_{|\beta|}} .
$$

Now putting together (4.34), (4.35) and (4.43), we see that

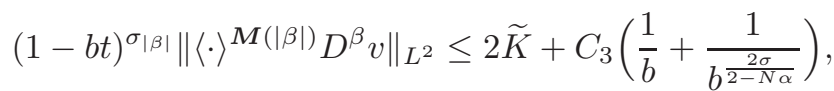

for $J-1 \leq|\beta| \leq J$. Therefore, if $b_{1} \geq b_{0}$ is sufficiently large so that

$$
C_{3}\left(\frac{1}{b}_{1}+\frac{1}{b_{1}^{\frac{2 \sigma}{2-N \alpha}}}\right) \leq \widetilde{K}
$$

then

$$
\sup _{J-1 \leq|\beta| \leq J} \sup _{0 \leq t \leq T^{*}}(1-b t)^{\sigma|\beta|}\left\|\langle\cdot\rangle^{M(|\beta|)} D^{\beta} v\right\|_{L^{2}} \leq 3 \widetilde{K}
$$

provided $b \geq b_{1}$.

STEP 4. Control of $\Phi_{3, T^{*}}$. It follows from (3.31) that

$$
\begin{aligned}
\frac{(1-b t)^{\frac{2-N \alpha}{2}}}{\inf _{x \in \mathbb{R}^{N}}\left(\langle x\rangle^{\alpha n}|v(t, x)|^{\alpha}\right)} \leq & \left(\inf _{x \in \mathbb{R}^{N}}\left(\langle x\rangle^{\alpha n}\left|v_{0}(x)\right|^{\alpha}\right)\right)^{-1}+\frac{2 \alpha|\Im \lambda|}{b(2-N \alpha)} \\
& +\alpha(1-b t)^{\frac{2-N \alpha}{2}} \int_{0}^{t}\left(\langle x\rangle^{n}|v|\right)^{-\alpha} \frac{|L(s, x)|}{|v|} d s .
\end{aligned}
$$

On the other hand, it follows from (4.14), (3.14), (3.29) and (3.15) that

$$
\left(\langle x\rangle^{n}|v|\right)^{-\alpha} \frac{|L(s, x)|}{|v|} \leq \widetilde{K}^{\alpha+1}(1-b s)^{-\frac{2-N \alpha}{2}-2 \sigma} .
$$

Since $\frac{2-N \alpha}{2}+2 \sigma<1$ by (3.5), we deduce that

Thus we see that

$$
\int_{0}^{t}\left(\langle x\rangle^{n}|v|\right)^{-\alpha} \frac{|L(s, x)|}{|v|} d s \leq \frac{C_{4}}{b} .
$$

$$
\frac{(1-b t)^{\frac{2-N \alpha}{2}}}{\inf _{x \in \mathbb{R}^{N}}\left(\langle x\rangle^{\alpha n}|v(t, x)|^{\alpha}\right)} \leq \widetilde{K}^{\alpha}+\frac{C_{4}}{b}
$$


Therefore, if $b_{1} \geq b_{0}$ is sufficiently large so that

$$
\frac{C_{4}}{b_{1}} \leq\left(2^{\alpha}-1\right) \widetilde{K}^{\alpha}
$$

then

$$
\Phi_{3, T^{*}} \leq 2 \widetilde{K}
$$

provided $b \geq b_{1}$.

SteP 5. Control of $\Phi_{4, T^{*}}$. The case $\beta=0$ is trivial, so we assume $|\beta| \geq 1$. Applying (2.6) with $f(t)=\lambda(1-b t)^{-\frac{4-N \alpha}{2}}|v|^{\alpha} v$ and $n=0$, and (3.28), we obtain

$$
\begin{aligned}
\frac{\partial}{\partial t}\left(\frac{\left|D^{\beta} v\right|}{|v|}\right)= & |v|^{-1} \frac{\partial}{\partial t}\left|D^{\beta} v\right|-|v|^{-2}\left|D^{\beta} v\right| \frac{\partial}{\partial t}|v| \\
= & -\left(|v|\left|D^{\beta} v\right|\right)^{-1} \Im\left(D^{\beta} \Delta v D^{\beta} \bar{v}\right)-|v|^{-2}\left|D^{\beta} v\right| L \\
& +(1-b t)^{-\frac{4-N \alpha}{2}}\left(|v|\left|D^{\beta} v\right|\right)^{-1} \Im\left(\lambda D^{\beta}\left(|v|^{\alpha} v\right) D^{\beta} \bar{v}\right) \\
& -(1-b t)^{-\frac{4-N \alpha}{2}}|v|^{\alpha-1}\left|D^{\beta} v\right| \Im \lambda .
\end{aligned}
$$

We first use a cancellation. Applying formula (4.36), we see that

$$
\begin{aligned}
& \left(|v|\left|D^{\beta} v\right|\right)^{-1} \Im\left(\lambda D^{\beta}\left(|v|^{\alpha} v\right) D^{\beta} \bar{v}\right)-|v|^{\alpha-1}\left|D^{\beta} v\right| \Im \lambda \\
& \quad=\left(|v|\left|D^{\beta} v\right|\right)^{-1} \Im\left(\lambda\left[D^{\beta}\left(|v|^{\alpha}\right) v+\lambda c_{\gamma_{1}, \gamma_{2}} \sum_{\substack{\gamma_{1}+\gamma_{2}=\beta \\
\left|\gamma_{1}\right|,\left|\gamma_{2}\right| \geq 1}} D^{\gamma_{1}}\left(|v|^{\alpha}\right) D^{\gamma_{2}} v\right] D^{\beta} \bar{v}\right)
\end{aligned}
$$

so that

$$
\left(|v|\left|D^{\beta} v\right|\right)^{-1} \Im\left(\lambda D^{\beta}\left(|v|^{\alpha} v\right) D^{\beta} \bar{v}\right)-|v|^{\alpha-1}\left|D^{\beta} v\right| \Im \lambda \leq \boldsymbol{G}
$$

where

$$
\boldsymbol{G}=|\lambda|\left|D^{\beta}\left(|v|^{\alpha}\right)\right|+C_{5} \sum_{\substack{\gamma_{1}+\gamma_{2}=\beta \\\left|\gamma_{1}\right|,\left|\gamma_{2}\right| \geq 1}} \frac{\left|D^{\gamma_{1}}\left(|v|^{\alpha}\right)\right|\left|D^{\gamma_{2}} v\right|}{|v|}
$$

Therefore, (4.48) yields

$$
\frac{\partial}{\partial t}\left(\frac{\left|D^{\beta} v\right|}{|v|}\right) \leq \frac{\left|D^{\beta} \Delta v\right|}{|v|}+\frac{\left|D^{\beta} v\right||\Delta v|}{|v|^{2}}+(1-b t)^{-\frac{4-N \alpha}{2}} \boldsymbol{G} .
$$

Integrating in $t$ and taking the sup in $x$, we deduce that

$$
\begin{aligned}
\left\|\frac{D^{\beta} v}{|v|}\right\|_{L^{\infty}} \leq & \widetilde{K}+\int_{0}^{t}\left(\left\|\frac{D^{\beta} \Delta v}{|v|}\right\|_{L^{\infty}}+\left\|\frac{D^{\beta} v}{|v|}\right\|_{L^{\infty}}\left\|\frac{\Delta v}{|v|}\right\|_{L^{\infty}}\right) \\
& +\int_{0}^{t}(1-b s)^{-\frac{4-N \alpha}{2}}\|\boldsymbol{G}\|_{L^{\infty}} .
\end{aligned}
$$

We first apply (4.14), (3.15) and the property $2 \sigma<1$ (by the fourth inequality in $(3.2))$, and we obtain

$$
\begin{aligned}
\int_{0}^{t}\left\|\frac{D^{\beta} v}{|v|}\right\|_{L^{\infty}}\left\|\frac{\Delta v}{|v|}\right\|_{L^{\infty}} & \leq C_{5} \int_{0}^{t}(1-b s)^{-\sigma_{|\beta|}-2 \sigma} \\
& \leq C_{5}(1-b t)^{-\sigma_{|\beta|}} \int_{0}^{t}(1-b s)^{-2 \sigma} \\
& \leq \frac{C_{5}}{b}(1-b t)^{-\sigma_{|\beta|}}
\end{aligned}
$$

Similarly, if $|\beta| \leq 2 m$, then (using also (3.7))

$$
\int_{0}^{t}\left\|\frac{D^{\beta} \Delta v}{|v|}\right\|_{L^{\infty}} \leq C_{5} \int_{0}^{t}(1-b s)^{-\sigma_{|\beta|}-2 \sigma} \leq \frac{C_{5}}{b}(1-b t)^{-\sigma_{|\beta|}} .
$$


For the case of $2 m+1 \leq|\beta| \leq 2 m+2$, we use Sobolev's embedding (2.14):

$$
\left\|\frac{D^{\beta} \Delta v}{|v|}\right\|_{L^{\infty}}=\left\|\frac{\langle\cdot\rangle^{n} D^{\beta} \Delta v}{\langle\cdot\rangle^{n}|v|}\right\|_{L^{\infty}} \leq C_{5}\left(\inf \langle x\rangle^{n}|v|\right)^{-1} \sum_{|\beta|+2 \leq|\gamma| \leq|\beta|+k}\left\|\langle\cdot\rangle^{n} D^{\gamma} v\right\|_{L^{2}} .
$$

It follows from (4.14), (3.13), (3.14) and (3.7) that

$$
\left\|\frac{D^{\beta} \Delta v}{|v|}\right\|_{L^{\infty}} \leq C_{5}(1-b s)^{-\frac{2-N \alpha}{2 \alpha}-(|\beta|+k+3) \sigma} .
$$

Since

$$
\frac{2-N \alpha}{2 \alpha}+(k+2) \sigma<1
$$

by the last inequality in (3.2), we deduce that

$$
\int_{0}^{t}\left\|\frac{D^{\beta} \Delta v}{|v|}\right\|_{L^{\infty}} \leq \frac{C_{5}}{b}(1-b t)^{-(|\beta|+1) \sigma} \leq \frac{C_{5}}{b}(1-b t)^{-\sigma_{|\beta|}} .
$$

Thus we see that for every $|\beta| \leq 2 m+2$,

$$
\int_{0}^{t}\left\|\frac{D^{\beta} \Delta v}{|v|}\right\|_{L^{\infty}} \leq \frac{C_{5}}{b}(1-b t)^{-\sigma_{|\beta|}} .
$$

We now estimate the last term in (4.50). We first assume $|\beta| \leq 2 m$, and we note that

$$
|\boldsymbol{G}| \leq C_{5} \sum_{\substack{\gamma_{1}+\gamma_{2}=\beta \\\left|\gamma_{1}\right| \geq 1}} \frac{\left|D^{\gamma_{1}}\left(|v|^{\alpha}\right)\right|\left|D^{\gamma_{2}} v\right|}{|v|}
$$

Since $1 \leq\left|\gamma_{1}\right| \leq 2 m$, we may apply (3.25) with $\beta$ replaced by $\gamma_{1}$. Using also (4.14) and (3.15), we see that

$$
\begin{aligned}
\left\|\frac{D^{\gamma_{1}}\left(|v|^{\alpha}\right) D^{\gamma_{2}} v}{|v|}\right\|_{L^{\infty}} & \leq\left\|D^{\gamma_{1}}\left(|v|^{\alpha}\right)\right\|_{L^{\infty}}\left\|\frac{D^{\gamma_{2}} v}{|v|}\right\|_{L^{\infty}} \\
& \leq C_{5} \min \left\{1,(b G(t))^{1-\frac{2 \sigma}{2-N \alpha}}\right\}(1-b t)^{-\left(\left|\gamma_{1}\right|-1\right) \sigma-\left|\gamma_{2}\right| \sigma} \\
& \leq C_{5} \min \left\{1,(b G(t))^{1-\frac{2 \sigma}{2-N \alpha}}\right\}(1-b t)^{-\sigma_{|\beta|}+\sigma} .
\end{aligned}
$$

Applying (4.6) of Lemma 4.2 with $\nu=\sigma_{|\beta|}$, we deduce that

$$
\int_{0}^{t}(1-b s)^{-\frac{4-N \alpha}{2}}\|\boldsymbol{G}\|_{L^{\infty}} \leq \frac{C_{5}}{b^{\frac{2 \sigma}{2-N \alpha}}}(1-b t)^{-\sigma_{|\beta|}} .
$$

We now consider the case $2 m+1 \leq|\beta| \leq 2 m+2$. Using (2.15) with $\rho=\alpha$,

$$
\left|D^{\beta}\left(|v|^{\alpha}\right)\right| \leq \alpha|v|^{\alpha} \frac{\left|D^{\beta} v\right|}{|v|}+C|v|^{\alpha} \sup _{\substack{0 \leq\left|\beta_{\ell}\right| \leq|\beta|-1 \\ \sum \beta_{\ell}=\beta}} \prod_{\ell=1}^{|\beta|} \frac{\left|D^{\beta_{\ell}} v\right|}{|v|} .
$$

Moreover, using (2.15) with $\rho=\alpha$ and $\beta$ replaced by $\gamma_{1}$,

$$
\left|D^{\gamma_{1}}\left(|v|^{\alpha}\right)\right| \leq C|v|^{\alpha} \sup _{\substack{0 \leq\left|\beta_{\ell}\right| \leq\left|\gamma_{1}\right| \\ \sum \beta_{\ell}=\gamma_{1}}} \prod_{\ell=1}^{|\beta|} \frac{\left|D^{\beta_{\ell}} v\right|}{|v|} .
$$

The two above inequalities imply

so that

$$
|\boldsymbol{G}| \leq|\lambda| \alpha|v|^{\alpha} \frac{\left|D^{\beta} v\right|}{|v|}+C_{5}|v|^{\alpha} \sum_{\substack{0 \leq\left|\beta_{\ell}\right| \leq|\beta|-1 \\ \sum \beta_{\ell}=\beta}} \prod_{\ell=1}^{|\beta|} \frac{\left|D^{\beta_{\ell}} v\right|}{|v|},
$$

$$
\int_{0}^{t}(1-b s)^{-\frac{4-N \alpha}{2}}\|\boldsymbol{G}\|_{L^{\infty}} \leq \widetilde{I}_{1}+\widetilde{I}_{2}
$$


where

and

$$
\widetilde{I}_{1}=|\lambda| \alpha \int_{0}^{t}(1-b s)^{-\frac{4-N \alpha}{2}}|v|^{\alpha} \frac{\left|D^{\beta} v\right|}{|v|}
$$

$$
\widetilde{I}_{2}=C_{5} \int_{0}^{t}(1-b s)^{-\frac{4-N \alpha}{2}}|v|^{\alpha} \sum_{\substack{0 \leq\left|\beta_{\ell}\right| \leq|\beta|-1 \\ \sum \beta_{\ell}=\beta}} \prod_{\ell=1}^{|\beta|} \frac{\left|D^{\beta_{\ell}} v\right|}{|v|} .
$$

We estimate $\widetilde{I}_{1}$ given by (4.56). Using (4.15), (4.14) and (3.15),

$$
|\lambda| \alpha|v|^{\alpha} \frac{\left|D^{\beta} v\right|}{|v|} \leq 5 \widetilde{K}|\lambda|\left(\frac{1+\alpha|\Im \lambda|}{|\Im \lambda|}\right) \min \left\{2 K^{\alpha}, b G(t)\right\}(1-b s)^{-\sigma_{|\beta|}} .
$$

Applying (4.7) of Lemma 4.2 with $\nu=\sigma_{|\beta|}$ and $M=2 K^{\alpha}$, and then (3.8), we deduce as in (4.39) that

$$
\widetilde{I}_{1} \leq\left(\frac{C_{5}}{b^{\frac{2 \sigma}{2-N \alpha}}}+\widetilde{K}\right)(1-b t)^{-\sigma_{|\beta|}} .
$$

Finally, we consider $\widetilde{I}_{2}$ given by (4.57). We estimate $|v|^{\alpha}$ by (3.22) and the terms $\frac{\left|D^{\beta} \ell v\right|}{|v|}$ by (4.14) and (3.15), and we obtain

$$
\left\||v|^{\alpha} \prod_{\ell=1}^{|\beta|} \frac{\left|D^{\beta_{\ell}} v\right|}{|v|}\right\|_{L^{\infty}} \leq C_{5} \min \{1, b G(t)\}(1-b t)^{-\sum_{\ell=1}^{|\beta|} \sigma_{\left|\beta_{\ell}\right|} .}
$$

Since $\sum_{\ell=1}^{|\beta|}\left|\beta_{\ell}\right|=|\beta|,\left|\beta_{\ell}\right| \leq|\beta|-1$ and $2 m+1 \leq|\beta| \leq 2 m+2$, it follows from (3.7) that

$$
\sum_{\ell=1}^{|\beta|} \sigma_{\left|\beta_{\ell}\right|} \leq \sigma_{|\beta|}-\sigma
$$

Moreover, $\min \{1, b G(t)\} \leq \min \left\{1,(b G(t))^{1-\frac{2 \sigma}{2-N \alpha}}\right\}$, so that

$$
\left\||v|^{\alpha} \prod_{\ell=1}^{|\beta|} \frac{\left|D^{\beta \ell} v\right|}{|v|}\right\|_{L^{\infty}} \leq C_{5} \min \left\{1,(b G(t))^{1-\frac{2 \sigma}{2-N \alpha}}\right\}(1-b t)^{-\sigma_{|\beta|}+\sigma} .
$$

Therefore, we deduce from estimate (4.6) of Lemma 4.2 with $\nu=\sigma_{|\beta|}$ that

$$
\widetilde{I}_{2} \leq \frac{C_{5}}{b^{\frac{2 \sigma}{2-N \alpha}}}(1-b t)^{-\sigma_{|\beta|}} .
$$

Estimates (4.54), (4.55), (4.58) and (4.59) show that

$$
\int_{0}^{t}(1-b s)^{-\frac{4-N \alpha}{2}}\|\boldsymbol{G}\|_{L^{\infty}} \leq\left(\frac{C_{5}}{b^{\frac{2 \sigma}{2-N \alpha}}}+\widetilde{K}\right)(1-b t)^{-\sigma_{|\beta|}},
$$

for all $|\beta| \leq 2 m+2$. Now we deduce from (4.50), (4.51), (4.53) and (4.60) that if $b_{1} \geq b_{0}$ is sufficiently large so that

$$
C_{5}\left(\frac{1}{b}+\frac{1}{b_{1}^{\frac{2 \sigma}{2-N \alpha}}}\right) \leq \widetilde{K}
$$

then

$$
\Phi_{4, T^{*}} \leq 2 \widetilde{K}
$$

provided $b \geq b_{1}$.

SteP 6. Conclusion. We choose $b_{1} \geq b_{0}$ sufficiently large so that (4.24), (4.32), (4.44), (4.46) and (4.61) are satisfied. It follows from (4.25), (4.33), (4.45), (4.47) and (4.62) that if $b \geq b_{1}$, then

$$
\Psi_{T^{*}} \leq 3 \widetilde{K}
$$

This contradicts (4.14) and completes the proof. 


\section{Asymptotics for $\left(\mathrm{NLS}_{b}\right)$ AND PRoOF of Theorem 1.1}

In this section, we establish time-asymptotic estimates for the solutions of $\left(\mathrm{NLS}_{b}\right)$ given by Proposition 4.1, which we use to prove Theorem 1.1. The asymptotic estimates are given by the following result.

Proposition 5.1. Let $\lambda \in \mathbb{C}$ satisfy $\Im \lambda<0$. Assume (1.4), (1.5)-(1.7), (3.2) and let $\mathcal{X}$ be defined by (1.10). Let $v_{0} \in \mathcal{X}$ satisfy (3.18) for some $K \geq 1$, let $b>b_{1}$ where $b_{1}$ is given by Proposition 4.1 , and let $v \in C\left(\left[0, \frac{1}{b}\right), \mathcal{X}\right)$ be the solution of $\left(\mathrm{NLS}_{b}\right)$ given by Proposition 4.1. It follows that there exist $C, \delta>0, \eta>\frac{N}{2}$ and $f_{0}, \omega_{0} \in L^{\infty}\left(\mathbb{R}^{N}\right) \cap C\left(\mathbb{R}^{N}\right)$ with $\left\|f_{0}\right\|_{L^{\infty}} \leq \frac{1}{2}$ and $\langle\cdot\rangle^{n} \omega_{0} \in L^{\infty}\left(\mathbb{R}^{N}\right)$ such that

$$
\left\|\langle\cdot\rangle^{\eta}\left[v(t, \cdot)-\omega_{0}(\cdot) \psi(t, \cdot) e^{-i \theta(t, \cdot)}\right]\right\|_{L^{\infty}} \leq C(1-b t)^{-\frac{2-N \alpha}{2 \alpha}-\delta},
$$

for all $0 \leq t<\frac{1}{b}$, where

$$
\psi(t, x)=\left(\frac{1+f_{0}(x)}{1+f_{0}(x)+\frac{2 \alpha|\Im \lambda|}{b(2-N \alpha)}\left|v_{0}(x)\right|^{\alpha}\left[(1-b t)^{-\frac{2-N \alpha}{2}}-1\right]}\right)^{\frac{1}{\alpha}}
$$

and

Moreover,

$$
\theta(t, x)=\frac{\Re \lambda}{\Im \lambda} \log \psi(t, x)
$$

In addition,

$$
\left|\omega_{0}\right|^{\alpha}=\frac{\left|v_{0}\right|^{\alpha}}{1+f_{0}}
$$

$$
(1-b t)^{-\frac{2-N \alpha}{2}}\|v\|_{L^{\infty}}^{\alpha} \underset{t \uparrow \frac{1}{b}}{\longrightarrow} \frac{b(2-N \alpha)}{2 \alpha|\Im \lambda|}
$$

and there exist two constants $0<a \leq A<\infty$ such that

$$
a(1-b t)^{\left(\frac{1}{\alpha}-\frac{N}{2}\right)\left(1-\frac{N}{2 n}\right)} \leq\|v(t)\|_{L^{2}} \leq A(1-b t)^{\left(\frac{1}{\alpha}-\frac{N}{2}\right)\left(1-\frac{N}{2 n}\right)},
$$

for all $0 \leq t<\frac{1}{b}$.

Proof. Using the a priori estimates of Proposition 4.1, we follow the proof of $[2$, Proposition 4.1].

We recall that (4.2) and (4.3) hold. Also, since $b_{1} \geq b_{0}$ of Proposition 3.2, $v$ satisfies (3.32) and (3.22). In particular

$$
|v(t, x)| \leq C \min \left\{\langle x\rangle^{-n},(1-b t)^{\frac{2-N \alpha}{2 \alpha}}\right\},
$$

for all $x \in \mathbb{R}^{N}$ and $\frac{1}{2 b} \leq t<\frac{1}{b}$. We let $f \in C\left(\left(0, \frac{1}{b}\right) \times \mathbb{R}^{N}\right)$ be defined by (3.34). It follows from $(4.2),(4.3),(3.37)$ with $K_{1}$ replaced by $5 \widetilde{K}$, and (3.38) that

$$
\|f(t, \cdot)-f(s, \cdot)\|_{L^{\infty}\left(\mathbb{R}^{N}\right)} \leq C \int_{t}^{s}(1-b \tau)^{-\frac{2-N \alpha}{2}-2 \sigma} d \tau \leq C(1-b t)^{1-\frac{2-N \alpha}{2}-2 \sigma},
$$

for all $0 \leq t<s<\frac{1}{b}$. Thus we see that $f(t, \cdot)$ is convergent in $L^{\infty}\left(\mathbb{R}^{N}\right)$ as $t \uparrow \frac{1}{b}$. Then $f$ can be extended to a continuous function $\left[0, \frac{1}{b}\right] \rightarrow L^{\infty}\left(\mathbb{R}^{N}\right)$. We set

$$
f_{0}(x)=f\left(\frac{1}{b}, x\right)=-\alpha \int_{0}^{\frac{1}{b}}\left|v_{0}(x)\right|^{\alpha}|v(s, x)|^{-\alpha-1} L(s, x) d s,
$$

so that $f_{0} \in L^{\infty}\left(\mathbb{R}^{N}\right) \cap C\left(\mathbb{R}^{N}\right)$ and

$$
\left\|f(t)-f_{0}\right\|_{L^{\infty}} \leq C(1-b t)^{1-\frac{2-N \alpha}{2}-2 \sigma} .
$$

Moreover, using (4.3),

$$
\|f(t)\|_{L^{\infty}} \leq \frac{1}{4}
$$


for all $0 \leq t \leq \frac{1}{b}$, and so

$$
\left\|f_{0}\right\|_{L^{\infty}} \leq \frac{1}{4}
$$

In particular, $1+f_{0}>0$, so that by $(5.2)$,

$$
0<\psi(t, x) \leq 1
$$

for all $0 \leq t<\frac{1}{b}$ and $x \in \mathbb{R}^{N}$. Moreover, it follows from (5.10) and (5.11) that

$$
\max \left\{\frac{1}{H}, \frac{1}{\widetilde{H}}\right\} \leq 2
$$

for all $0 \leq t<\frac{1}{b}$, where $H$ is defined by (3.33) and

$$
\widetilde{H}(t, x)=1+f_{0}(x)+\frac{2 \alpha|\Im \lambda|}{b(2-N \alpha)}\left|v_{0}(x)\right|^{\alpha}\left[(1-b t)^{-\frac{2-N \alpha}{2}}-1\right] .
$$

Note also that by (5.10), (5.11) and (3.18),

$$
\begin{aligned}
\langle x\rangle^{n \alpha} \min \{\tilde{H}, H\} & \geq \frac{2 \alpha|\Im \lambda|}{b(2-N \alpha)}\left(\langle x\rangle^{n}\left|v_{0}(x)\right|\right)^{\alpha}\left[(1-b t)^{-\frac{2-N \alpha}{2}}-1\right] \\
& \geq \frac{2 \alpha|\Im \lambda|}{b(2-N \alpha) K^{\alpha}}\left[(1-b t)^{-\frac{2-N \alpha}{2}}-1\right]
\end{aligned}
$$

so that

$$
\max \left\{\frac{1}{H}, \frac{1}{\widetilde{H}}\right\} \leq C(1-b t)^{\frac{2-N \alpha}{2}}\langle x\rangle^{n \alpha},
$$

for $\frac{1}{2 b} \leq t<\frac{1}{b}$. Moreover, it follows from (5.9) that

$$
\left\|\frac{\widetilde{H}}{H}-1\right\|_{L^{\infty}}=\left\|\frac{f(t)-f_{0}}{H}\right\|_{L^{\infty}} \leq C .
$$

We now set

$$
\widetilde{v}(t, x)=\left(\frac{\left|v_{0}(x)\right|^{\alpha}}{\widetilde{H}(t, x)}\right)^{\frac{1}{\alpha}} .
$$

It follows from (3.32), (5.16), (5.9), (5.13) and (5.14) that

$$
\left\||v(t, \cdot)|^{\alpha}-\widetilde{v}(t, \cdot)^{\alpha}\right\|_{L^{\infty}}=\left\|\left(\langle\cdot\rangle^{n}\left|v_{0}\right|\right)^{\alpha} \frac{f(t)-f_{0}}{\langle\cdot\rangle^{n \alpha} H \widetilde{H}}\right\|_{L^{\infty}} \leq C(1-b t)^{1-2 \sigma},
$$

and

$$
\begin{aligned}
\left\|\frac{|v(t, \cdot)|^{\alpha}-\widetilde{v}(t, \cdot)^{\alpha}}{\langle\cdot\rangle^{n \alpha}}\right\|_{L^{\infty}} & =\left\|\left(\langle\cdot\rangle^{n}\left|v_{0}\right|\right)^{\alpha} \frac{f(t)-f_{0}}{\langle\cdot\rangle^{2 n \alpha} H \widetilde{H}}\right\|_{L^{\infty}} \\
& \leq C(1-b t)^{1-2 \sigma+\frac{2-N \alpha}{2}},
\end{aligned}
$$

for all $\frac{2}{b} \leq t<\frac{1}{b}$. Therefore, given any $0 \leq \rho \leq 1$, we have

$$
\left\|\frac{|v(t, \cdot)|^{\alpha}-\widetilde{v}(t, \cdot)^{\alpha}}{\langle\cdot\rangle^{\rho n \alpha}}\right\|_{L^{\infty}} \leq C(1-b t)^{1-2 \sigma+\rho \frac{2-N \alpha}{2}}
$$

for all $\frac{2}{b} \leq t<\frac{1}{b}$. Next, we introduce the decomposition

$$
v(t, x)=\omega(t, x) \psi(t, x) e^{-i \theta(t, x)},
$$

where $\psi(t, x)$ and $\theta(t, x)$ are defined by (5.2) and (5.3) respectively. Differentiating (5.18) with respect to $t$, we obtain

$$
i \partial_{t} \omega=i \frac{e^{i \theta}}{\psi} \partial_{t} v-i \omega \frac{\partial_{t} \psi}{\psi}-\omega \partial_{t} \theta
$$


On the other hand, it follows easily from (5.2), (5.3) and (5.16) that

$$
\begin{aligned}
& \frac{\partial_{t} \psi}{\psi}=\Im \lambda(1-b t)^{-\frac{4-N \alpha}{2}} \widetilde{v}^{\alpha}, \\
& \partial_{t} \theta=\Re \lambda(1-b t)^{-\frac{4-N \alpha}{2}} \widetilde{v}^{\alpha} .
\end{aligned}
$$

Therefore, we deduce from (5.19), (5.18) and $\left(\mathrm{NLS}_{b}\right)$ that

$$
\begin{aligned}
i \partial_{t} \omega & =i \frac{e^{i \theta}}{\psi} \partial_{t} v-\omega(1-b t)^{-\frac{4-N \alpha}{2}} \lambda \widetilde{v}^{\alpha} \\
& =\frac{e^{i \theta}}{\psi}\left(i \partial_{t} v-\lambda(1-b t)^{-\frac{4-N \alpha}{2}} \widetilde{v}^{\alpha} v\right) \\
& =\frac{e^{i \theta}}{\psi}\left(-\Delta v+\lambda(1-b t)^{-\frac{4-N \alpha}{2}}\left(|v|^{\alpha}-\widetilde{v}^{\alpha}\right) v\right) .
\end{aligned}
$$

We claim that there exists $\eta>0$ such that

$$
\begin{gathered}
0 \leq \eta \leq n, \\
\eta \geq n(1-\alpha), \\
\eta>\frac{N}{2}, \\
2 \sigma+\frac{2-N \alpha}{2}\left[\frac{\eta+n \alpha}{n \alpha}\right]<1 .
\end{gathered}
$$

Indeed, if $\alpha \geq \frac{4}{5}$, we let $\eta=\frac{n}{5}$. Conditions (5.21) and (5.22) are obviously satisfied, and (5.23) follows from the first inequality in (3.1). Moreover, (5.24) is equivalent to

$$
2 \sigma<\frac{N}{2}\left(\alpha+\frac{1}{5}\right)-\frac{1}{5 \alpha}
$$

Since $\alpha \geq \frac{4}{5}$ and $N \geq 1$, the right-hand side of the above inequality is $\geq \frac{1}{4}$. Since $\sigma<\frac{1}{8}$ by the fourth inequality in (3.2) and (1.5) we see that (5.24) is satisfied. If $\alpha<\frac{4}{5}$, we let $\eta=n(1-\alpha)$, so that (5.21) and (5.22) hold. Moreover, $\eta \geq \frac{n}{5}$ so that (5.23) follows from the first inequality in (3.1). Furthermore, (5.24) is equivalent to $\sigma<\frac{(N+2) \alpha-2}{4 \alpha}$, which is a consequence of the last inequality in (3.2).

We fix $\eta>0$ satisfying (5.21)-(5.24) and we let

$$
\delta=1-\frac{2-N \alpha}{2 \alpha}-2 \sigma-\frac{2-N \alpha}{2}\left[\frac{\eta-n(1-\alpha)}{n \alpha}\right]>0 .
$$

It follows from (5.20) that

$$
\begin{aligned}
& \left\|\langle\cdot\rangle^{\eta} \partial_{t} \omega\right\|_{L^{\infty}} \\
& \quad \leq\left\|\langle\cdot\rangle^{n} \frac{v}{\psi}\right\|_{L^{\infty}}\left(\left\|\frac{\Delta v}{\langle\cdot\rangle^{n-\eta}|v|}\right\|_{L^{\infty}}+|\lambda|(1-b t)^{-\frac{4-N \alpha}{2}}\left\|\frac{|v|^{\alpha}-\widetilde{v}^{\alpha}}{\langle\cdot\rangle^{n-\eta}}\right\|_{L^{\infty}}\right) .
\end{aligned}
$$

Applying (3.18) (3.32) (5.2), (5.11) and (5.15), we obtain

$$
\left\|\langle\cdot\rangle^{n} \frac{v}{\psi}\right\|_{L^{\infty}}=\left\|\langle\cdot\rangle^{n} v_{0}\left(\frac{1}{1+f_{0}} \frac{\widetilde{H}}{H}\right)^{\frac{1}{\alpha}}\right\|_{L^{\infty}} \leq C .
$$

Moreover, by (4.2) and (3.15),

$$
\left\|\frac{\Delta v}{\langle\cdot\rangle^{n-\eta}|v|}\right\|_{L^{\infty}} \leq\left\|\frac{\Delta v}{|v|}\right\|_{L^{\infty}} \leq 5 \widetilde{K}(1-b t)^{-2 \sigma} .
$$

Furthermore, since $0 \leq n-\eta \leq n \alpha$, it follows from (5.17) with $\rho=\frac{n-\eta}{n \alpha}$ that

$$
\left\|\frac{|v|^{\alpha}-\widetilde{v}^{\alpha}}{\langle\cdot\rangle^{n-\eta}}\right\|_{L^{\infty}} \leq C(1-b t)^{1-2 \sigma+\frac{(n-\eta)(2-N \alpha)}{2 n \alpha}} .
$$


We deduce from (5.26)-(5.29) that

$$
\begin{aligned}
\left\|\langle\cdot\rangle^{\eta} \partial_{t} \omega\right\|_{L^{\infty}} & \leq C(1-b t)^{-2 \sigma}+C(1-b t)^{-2 \sigma-\frac{2-N \alpha}{2}\left[\frac{\eta-n(1-\alpha)}{n \alpha}\right]} \\
& \leq C(1-b t)^{-2 \sigma-\frac{2-N \alpha}{2}\left[\frac{\eta-n(1-\alpha)}{n \alpha}\right]} .
\end{aligned}
$$

Applying (5.24) and (5.25), we obtain

$$
\left\|\langle\cdot\rangle^{\eta}(\omega(t)-\omega(s))\right\|_{L^{\infty}} \leq C(1-b t)^{1-2 \sigma-\frac{2-N \alpha}{2}\left[\frac{\eta-n(1-\alpha)}{n \alpha}\right]}=C(1-b t)^{\frac{2-N \alpha}{2 \alpha}+\delta},
$$

for all $\frac{1}{2 b} \leq t<s<\frac{1}{b}$. We conclude that there exists $\omega_{0} \in L^{\infty}\left(\mathbb{R}^{N}\right) \cap C\left(\mathbb{R}^{N}\right)$ such that $\langle x\rangle^{\eta} \omega_{0} \in L^{\infty}\left(\mathbb{R}^{N}\right)$ and

$$
\left\|\langle\cdot\rangle^{\eta}\left(\omega(t)-\omega_{0}\right)\right\|_{L^{\infty}} \leq C(1-b t)^{\frac{2-N \alpha}{2 \alpha}+\delta},
$$

for all $\frac{1}{2 b} \leq t<\frac{1}{b}$. Moreover, (5.18) and (5.27) imply that $\left\|\langle\cdot\rangle^{n} \omega(t)\right\|_{L^{\infty}} \leq C$, so that $\langle\cdot\rangle^{n} \omega_{0} \in L^{\infty}\left(\mathbb{R}^{N}\right)$. Applying (5.18), (5.12) and (5.31), we obtain

$$
\left\|\langle\cdot\rangle^{\eta}\left(v(t)-\omega_{0} \psi e^{-i \theta}\right)\right\|_{L^{\infty}}=\left\|\langle\cdot\rangle^{\eta} \psi\left(\omega(t)-\omega_{0}\right)\right\|_{L^{\infty}} \leq C(1-b t)^{\frac{2-N \alpha}{2 \alpha}+\delta},
$$

for all $\frac{1}{2 b} \leq t<\frac{1}{b}$. This proves the asymptotic estimate (5.1) for $\frac{1}{2 b} \leq t<\frac{1}{b}$. For $0 \leq t \leq \frac{1}{2 b}$ it is clearly satisfied by possibly choosing $C$ larger.

Now, we prove (5.4). It follows from (5.17) and (5.18) that

$$
\left\||\omega(t)|^{\alpha} \psi(t)^{\alpha}-\widetilde{v}(t)^{\alpha}\right\|_{L^{\infty}} \leq C(1-b t)^{1-2 \sigma} .
$$

Using (5.2) and (5.16), we deduce that

$$
\left\|\frac{|\omega(t)|^{\alpha}\left(1+f_{0}\right)-\left|v_{0}\right|^{\alpha}}{\widetilde{H}}\right\|_{L^{\infty}} \leq C(1-b t)^{1-2 \sigma} .
$$

Since by (3.18) and (5.11)

$$
\widetilde{H} \leq C(1-b t)^{-\frac{2-N \alpha}{2}}
$$

we conclude that

$$
\left\||\omega(t)|^{\alpha}\left(1+f_{0}\right)-\left|v_{0}\right|^{\alpha}\right\|_{L^{\infty}} \leq C(1-b t)^{1-\frac{2-N \alpha}{2}-2 \sigma \underset{t \rightarrow \frac{1}{b}}{\longrightarrow} 0 .} .
$$

Applying (5.31), we obtain property (5.4).

Next we prove (5.5). Set

$$
Z(t, x)=(1-b t)^{-\frac{2-N \alpha}{2}} \widetilde{v}(t, x)^{\alpha} .
$$

It follows from (5.16) that

$$
Z(t, x)=\frac{\left|v_{0}(x)\right|^{\alpha}(1-b t)^{-\frac{2-N \alpha}{2}}}{1+f_{0}(x)+\frac{2 \alpha|\Im \lambda|}{b(2-N \alpha)}\left|v_{0}(x)\right|^{\alpha}\left[(1-b t)^{-\frac{2-N \alpha}{2}}-1\right]} .
$$

Since $1+f_{0} \geq 0$ by (5.11), we obtain

$$
Z(t, x) \leq \frac{b(2-N \alpha)}{2 \alpha|\Im \lambda|} \frac{1}{1-(1-b t)^{\frac{2-N \alpha}{2}}}
$$

so that

$$
\limsup _{t \uparrow \frac{1}{b}}\|Z(t)\|_{L^{\infty}} \leq \frac{b(2-N \alpha)}{2 \alpha|\Im \lambda|}
$$

Moreover, $1+f_{0} \leq 2$, so that

$$
Z(t, 0) \geq \frac{\left|v_{0}(0)\right|^{\alpha}(1-b t)^{-\frac{2-N \alpha}{2}}}{2+\frac{2 \alpha|\Im \lambda|}{b(2-N \alpha)}\left|v_{0}(0)\right|^{\alpha}\left[(1-b t)^{-\frac{2-N \alpha}{2}}-1\right]}
$$


Since $\left|v_{0}(0)\right|>0$ by (3.18), we deduce that

$$
\liminf _{t \uparrow \frac{1}{b}}\|Z(t)\|_{L^{\infty}} \geq \frac{b(2-N \alpha)}{2 \alpha|\Re \lambda|}
$$

Thus we see that

$$
\|Z(t)\|_{L^{\infty}} \underset{t \rightarrow \frac{1}{b}}{\longrightarrow} \frac{b(2-N \alpha)}{2 \alpha|\Re \lambda|} .
$$

Applying (5.34) and (5.17), we deduce that

$$
\begin{aligned}
\left\|(1-b t)^{-\frac{2-N \alpha}{2}}|v(t)|^{\alpha}-Z(t)\right\|_{L^{\infty}} & =(1-b t)^{-\frac{2-N \alpha}{2}}\left\||v(t)|^{\alpha}-\widetilde{v}(t)^{\alpha}\right\|_{L^{\infty}} \\
& \leq C(1-b t)^{1-(2-N \alpha)-2 \sigma \underset{t \rightarrow \frac{1}{b}}{\longrightarrow} 0,}
\end{aligned}
$$

so that (5.5) follows from (5.35).

Finally we prove (5.6), and we let $\frac{1}{2 b} \leq t<\frac{1}{b}$. It follows from (5.7) that

$$
\begin{aligned}
\int_{\mathbb{R}^{N}}|v(t)|^{2} & =\int_{\langle x\rangle>(1-b t)^{-\frac{2-N \alpha}{2 n \alpha}}}|v(t)|^{2}+\int_{\langle x\rangle<(1-b t)^{-\frac{2-N \alpha}{2 n \alpha}}}|v(t)|^{2} \\
& \leq C \int_{\langle x\rangle>(1-b t)^{-\frac{2-N \alpha}{2 n \alpha}}}\langle x\rangle^{-2 n}+C \int_{\langle x\rangle<(1-b t)^{-\frac{2-N \alpha}{2 n \alpha}}}(1-b t)^{\frac{2-N \alpha}{\alpha}} \\
& \leq C(1-b t)^{\frac{2-N \alpha}{\alpha}\left(1-\frac{N}{2 n}\right)},
\end{aligned}
$$

which proves the upper estimate in (5.6). Next, using (5.11), we see that

$$
\widetilde{H}^{\frac{1}{\alpha}} \leq \begin{cases}C, & \langle x\rangle>(1-b t)^{-\frac{2-N \alpha}{2 n \alpha}} \\ C\langle x\rangle^{-n}, & \langle x\rangle<(1-b t)^{-\frac{2-N \alpha}{2 n \alpha}} .\end{cases}
$$

Applying (3.32) and $\left|v_{0}(x)\right| \geq K^{-1}\langle x\rangle^{-n}$ (by (3.18)), we deduce that

$$
|v(t, x)| \geq \begin{cases}c\langle x\rangle^{-n}, & \langle x\rangle>(1-b t)^{-\frac{2-N \alpha}{2 n \alpha}} \\ c, & \langle x\rangle<(1-b t)^{-\frac{2-N \alpha}{2 n \alpha}}\end{cases}
$$

for some $c>0$. Therefore,

$$
\int_{\mathbb{R}^{N}}|v(t)|^{2} \geq c \int_{\langle x\rangle>(1-b t)^{-\frac{2-N \alpha}{2 n \alpha}}}\langle x\rangle^{-2 n}+c \int_{\langle x\rangle\left\langle(1-b t)^{-\frac{2-N \alpha}{2 n \alpha}}\right.}(1-b t)^{\frac{2-N \alpha}{\alpha}},
$$

from which the lower estimate in (5.6) follows. This completes the proof.

We are now in a position to prove Theorem 1.1.

Proof of Theorem 1.1. Let $v_{0} \in \mathcal{X}$ satisfy (1.11), and set

$$
K=\left\|v_{0}\right\|_{\mathcal{X}}+\left(\inf _{x \in \mathbb{R}^{N}}\langle x\rangle^{n}\left|v_{0}(x)\right|\right)^{-1} .
$$

Let $b \geq b_{1}$, where $b_{1}$ is given by Proposition 4.1 for this value of $K$, and let $v \in C\left(\left[0, \frac{1}{b}\right), \mathcal{X}\right)$ be the solution of $\left(\mathrm{NLS}_{b}\right)$ given by Proposition 4.1. Let $f_{0}, \omega_{0}, \theta, \psi$ be given by Proposition 5.1. Set

$$
u(t, x)=(1+b t)^{-\frac{N}{2}} e^{i \frac{b|x|^{2}}{4(1+b t)}} v\left(\frac{t}{1+b t}, \frac{x}{1+b t}\right), t \geq 0, x \in \mathbb{R}^{N} .
$$

It follows that $u \in C\left([0, \infty), H^{1}\left(\mathbb{R}^{N}\right)\right)$ is the solution of (NLS) with the initial value $u_{0}(x)=e^{i \frac{b|x|^{2}}{4}} v_{0}(x)$. Since $\eta>\frac{N}{2}$, the estimate (5.1) implies

$$
\left\|v(t, x)-\omega_{0}(x) \psi(t, x) e^{-i \theta(t, x)}\right\|_{L^{\infty} \cap L^{2}} \leq C(1-b t)^{\frac{2-N \alpha}{2 \alpha}+\delta} .
$$

Then, (1.12) follows from (5.1). By using (5.36), we see that (1.13) and (1.14) are consequences of (5.5) and (5.6), respectively. This completes the proof. 


\section{REFERENCES}

[1] T. Cazenave, S. Correia, F. Dickstein and F.B. Weissler: A Fujita-type blowup result and low energy scattering for a nonlinear Schrödinger equation. São Paulo J. Math. Sci. 9 (2015), no. 2, 146-161. (MR3457455) (doi: 10.1007/s40863-015-0020-6)

[2] T. Cazenave and Z. Han: Asymptotic behavior for a Schrödinger equation with nonlinear subcritical dissipation. Discrete Contin. Dynam. Systems 40 (2020), no. 8, 4801-4819. (doi: 10.3934/dcds.2020202)

[3] T. Cazenave, Z. Han and Y. Martel: Blowup on an arbitrary compact set for a Schrödinger equation with nonlinear source term. J. Dynam. Differential Equations (2020). (doi: 10.1007/s10884-020-09841-8)

[4] T. Cazenave, Y. Martel and L. Zhao: Finite-time blowup for a Schrödinger equation with nonlinear source term. Discrete Contin. Dynam. Systems 39 (2019), no. 2, 1171-1183. (MR3918212) (doi: 10.3934/dcds.2019050)

[5] T. Cazenave and I. Naumkin: Local existence, global existence, and scattering for the nonlinear Schrödinger equation. Commun. Contemp. Math. 19 (2017), no. 2, 1650038, 20 pp. (MR3611666) (doi: 10.1142/S0219199716500383)

[6] T. Cazenave and I. Naumkin: Modified scattering for the critical nonlinear Schrödinger equation. J. Funct. Anal. 274 (2018), no. 2, 402-432. (MR3724144) (doi: $10.1016 / j \cdot j f a .2017 .10 .022)$

[7] T. Cazenave and F. B. Weissler: Rapidly decaying solutions of the nonlinear Schrödinger equation, Comm. Math. Phys. 147 (1992), 75-100. (MR1171761) (doi: 10.1007/BF02099529)

[8] G.M. Constantine and T.H. Savits: A multivariate Faa di Bruno formula with applications, Trans. Amer. Math. Soc. 348 (1996), no.2, 503-520. (MR1325915) (doi: 10.1090/S0002-9947-96-01501-2)

[9] M.C. Cross and P.C. Hohenberg: Pattern formation outside of equilibrium, Rev. Mod. Phys. 65 (1993), no. 3, 851-1112. (doi: 10.1103/RevModPhys.65.851)

[10] J. Ginibre, T. Ozawa and G. Velo: On the existence of the wave operators for a class of nonlinear Schrödinger equations, Ann. Inst. H. Poincaré Phys. Théor. 60 (1994), no. 2, 211-239. (MR1270296) (link: http://archive.numdam.org/article/AIHPA_1994__60_2_211_0.pdf)

[11] J. Ginibre and G. Velo: On a class of nonlinear Schrödinger equations. II. Scattering theory, general case, J. Funct. Anal. 32, no. 1 (1979), 33-71. (MR0533219) (doi: 10.1016/0022-1236(79) 90077-6)

[12] J. Ginibre and G. Velo: On a class of nonlinear Schrödinger equations. III. Special theories in dimensions 1, 2 and 3, Ann. Inst. Henri Poincaré 28 (1978), 287-316. (MR0498408) (link: http://archive.numdam.org/article/AIHPA_1978__28_3_287_0.pdf)

[13] N. Hayashi, C. Li and P.I. Naumkin: Time decay for nonlinear dissipative Schrödinger equations in optical fields. Adv. Math. Phys. 2016, Art. ID 3702738, 7 pp. (MR3465033) (doi: 10.1155/2016/3702738)

[14] N. Hayashi, C. Li and P.I. Naumkin: Upper and lower time decay bounds for solutions of dissipative nonlinear Schrödinger equations. Commun. Pure Appl. Anal. 16 (2017), no. 6, 2089-2104. (MR3693873) (doi: 10.3934/cpaa.2017103)

[15] N. Kita and A. Shimomura: Asymptotic behavior of solutions to Schrödinger equations with a subcritical dissipative nonlinearity, J. Differential Equations 242 (2007), no. 1, 192-210. (MR2361107) (doi: 10.1016/j·jde.2007.07.003)

[16] N. Kita and A. Shimomura: Large time behavior of solutions to Schrödinger equations with a dissipative nonlinearity for arbitrarily large initial data. J. Math. Soc. Japan 61 (2009), no. 1, 39-64. (MR2272871) (doi: 10.2969/jmsj/06110039)

[17] A. Mielke: The Ginzburg-Landau equation in its role as a modulation equation. in Handbook of dynamical systems, Vol. 2, 759-834, North-Holland, Amsterdam, 2002. (MR1901066) (doi: 10.1016/S1874-575X (02)80036-4)

[18] K. Nakanishi and T. Ozawa: Remarks on scattering for nonlinear Schrödinger equations, NoDEA Nonlinear Differential Equations Appl. 9 (2002), no. 1, 45-68. (MR1891695) (doi: $10.1007 / \mathrm{s} 00030-002-8118-9$ )

[19] A. Shimomura: Asymptotic behavior of solutions for Schrödinger equations with dissipative nonlinearities, Comm. Partial Differential Equations 31 (2006), no. 7-9, 1407-1423. (MR2254620) (doi: 10.1080/03605300600910316)

[20] K. Stewartson and J.T. Stuart: A non-linear instability theory for a wave system in plane Poiseuille flow. J. Fluid Mech. 48 (1971), 529-545. (MR0309420) (doi: 10.1017/S0022112071001733)

[21] W.A. Strauss: Nonlinear scattering theory at low energy: sequel, J. Funct. Anal. 43 (1981), 281-293. (MR0614228) (doi: 10.1016/0022-1236 (81) 90063-X) 
E-mail address: thierry.cazenave@sorbonne-universite.fr

E-mail address: hanzh_0102@hznu.edu.cn

E-mail address: ivan.naumkin@iimas.unam.mx

${ }^{1}$ Sorbonne Université \& CNRS, Laboratoire Jacques-Louis Lions, B.C. 187, 4 Place Jussieu, 75252 Paris Cedex 05, France

${ }^{2}$ Department of Mathematics, Hangzhou Normal University, Hangzhou, 311121, China

${ }^{3}$ Departamento de Física Matemática, Instituto de Investigaciones en Matemáticas Aplicadas y en Sistemas. Universidad Nacional Autónoma de México, Apartado Postal 20-126, Ciudad de MÉxico, 01000, MÉxico. 